\title{
Review of Empoasca (Distantasca) Dworakowska (Hemiptera: Cicadellidae: Typhlocybinae: Empoascini), with description of two new species from China
}

\author{
YA-LIN ZHANG ${ }^{1}$, YANG LIU ${ }^{2} \&$ DAO-ZHENG QIN ${ }^{3}$ \\ Key Laboratory of Plant Protection Resources and Pest Management of Ministry of Education, Entomological Museum, Northwest \\ A\&F University, Yangling, Shaanxi 712100, China. E-mail: ${ }^{1}$ yalinzh@yahoo.com.cn; ${ }^{2}$ liuyangxnzb@hotmail.com \\ ${ }^{3}$ Corresponding author. E-mail: qindaozh0426@yahoo.com.cn
}

\begin{abstract}
A key to known species of the subgenus Empoasca (Distantasca) is provided. Two new species, E. (Distantasca) latibasis Zhang and Liu sp. n. and E. (D.) tuberculata Zhang and Liu sp. n., are described and illustrated from Yunnan Province (SW China).
\end{abstract}

Key words: Homoptera, Cicadomorpha, Empoasca (Distantasca), new species, taxonomy, China

\section{Introduction}

The leafhopper subgenus Empoasca (Distantasca) was first described as a separate genus by Dworakowska (1972), with Empoasca terminalis Distant (1918) as the type species. Dworakowska (1972) found Distantasca has the same structure and wings venation as in Empoasca Walsh, but male genital segments different. Then Distantasca was subsequently downgraded to a subgenus of Empoasca Walsh (Dworakowska \& Viraktamath, 1975). It is primarily characterized by the well developed anal tube appendages and the long and narrow subgenital plates with macrosetae not reaching the tip of the plate and two bands of long, hair-like setae on the lateral surface, one near the mid-length of the plate and one just before the apex, often separated by a few thin but short setae. Empoasca (Distantasca) has been studied previously by Dworakowska (1972, 1977, 1980, 1981, 1994), 10 new species have been illustrated in all, and Qin \& Zhang (2007) described 10 species, including 2 new species.

Up to now, 13 species have been reported world-wide. Most species of this subgenus are distributed in SE Asia and adjacent areas of the Palaearctic Region. In this paper, two new species, E. (Distantasca) latibasis and E. (Distantasca) tuberculata spp. n., are described and illustrated and a key to species of the subgenus is provided.

\section{Material and methods}

All the specimens examined, including types of the new species, are deposited in the Entomological Museum, Northwest A\&F University (NWAFU). Habitus photos were taken by using a Scientific Digital micrography system equipped with an Auto-montage imaging system and a high sensitive QIMAGING Retiga 4000R digital camera (CCD). Multiple photographs were compressed into final images. The body measurements are from apex of vertex to tip of forewing. The morphological terminology used in this description follows Zhang (1990) except for the nomenclature of the wing venation, for which we follow Dworakowska (1993). 


\section{Key to the worldwide species of Empoasca (Distantasca) (males)}

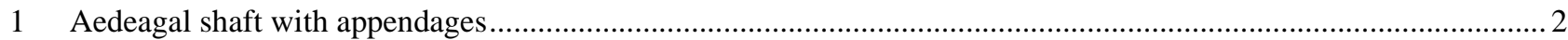

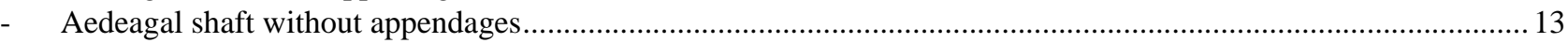

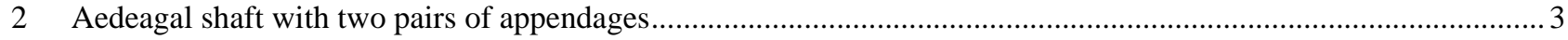

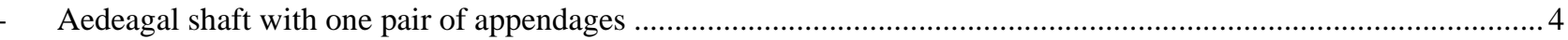

3 Anal tube appendages with large subapical tooth (Fig. 145)............................... (D.) paraterminalis Qin \& Zhang Anal tube appendages without large subapical tooth (Figs. 4, 5) ....................................... E. (D.) terminalis Distant

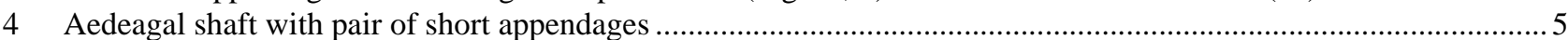

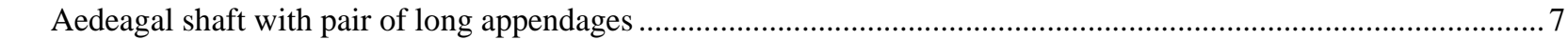

5 Anal tube appendages ornamented with small teeth apically (Fig. 61) ..........................E. (D.) latava Dworakowska

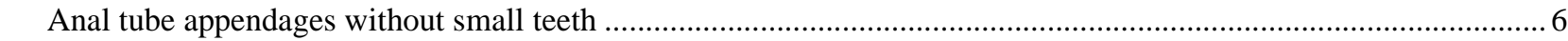

6 Anal tube appendages with large subapical tooth (Figs. 136, 137) ...................... (D.) latibasis Zhang \& Liu sp. n.

Anal tube appendages smooth, without large tooth (Fig. 154) ................................. (D.) serratipenis Qin \& Zhang

7 Aedeagal shaft appendages ornamented with small teeth apically (Figs. 121, 122)

E. (D.) tuberculata Zhang \& Liu sp. n.

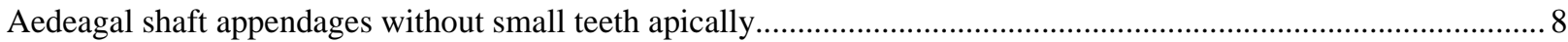

8 Aedeagal shaft and appendages equally broad in profile (Figs. 20, 22) .......................E. (D.) faciata Dworakowska

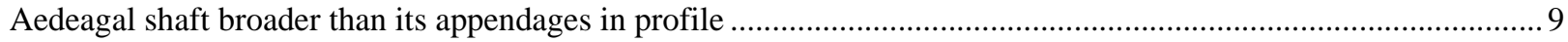

9 Aedeagal shaft with a pair of small lateral extensions consisting of small tubercles (Figs. 49, 50)

E. (D.) rabava Dworakowska

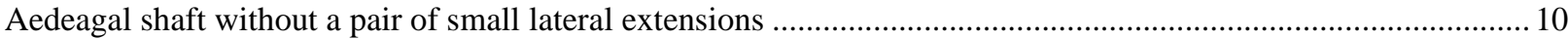

10 Anal tube appendages with large subapical tooth (Fig. 27) ........................................... (D.) riora Dworakowska

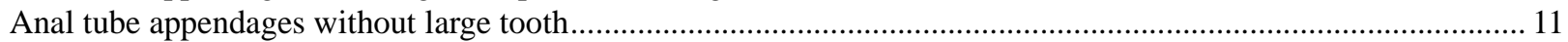

11 Anal tube appendages smooth, without small teeth (Figs. 110, 111)........................... E. (D.) bulbosa Dworakowska

Anal tube appendages not smooth, with small teeth (Figs. 41, 42, 55) …....................................................... 12

12 Aedeagus appendages curved mesad at about $1 / 3$ of their length from tip (Figs. 43, 45)....E. (D.) tna Dworakowska

- Aedeagus appendages not curved strongly mesad (Fig. 56)........................................... (D.) barawa Dworakowska

13 Aedeagal shaft with lamellate lateral extensions at ventral margin (Figs. 75, 78) .......... E. (D.) rokasa Dworakowska

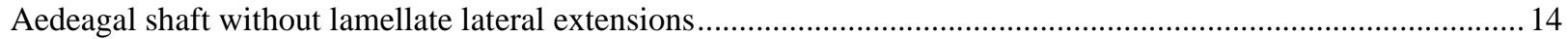

14 Aedeagal shaft strongly expanded ventrad in profile (Figs. 100, 102), anal tube appendages finely serrated at margins.

E. (D.) tiaca Dworakowska

- Aedeagal shaft only slightly expanded in profile (Figs. 86, 88), anal tube appendages smooth.

E. (D.) atika Dworakowska

\section{Empoasca (Distantasca) terminalis Distant, 1918}

(Figs. 1-12, 158, 171, 184, 197)

Empoasca terminalis Distant, 1918: 92.

Empoasca crenulata Linnavuori, 1960: 257.

Empoasca (Distantasca) terminalis, Dworakowska, 1972: 25; Dworakowska, 1981: 158; Qin \& Zhang, $2007: 191$.

Specimens examined: China: 1 , Liangyuan, Hainan Prov., 1 June 1983, coll. Zhang Yalin; $2 \curvearrowright$, Zhaoqing, Guangdong Prov., 20 June 1983, coll. Zhang Yalin; 1 đ̂, Tongshi, Hainan Prov., 7 June 1983, coll. Zhang

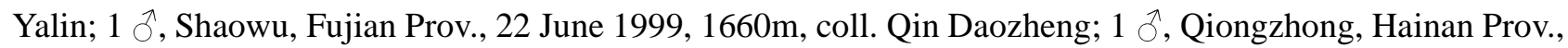
5 June 1983, coll. Zhang Yalin; 2 q, Yacheng, Hainan Prov., 8 June 1983, coll. Zhang Yalin; 1 ठ , Dinghushan in Zhaoqing, Guangdong Prov., 20 June 1983, coll. Zhang Yalin.

Distribution: China (Hainan, Fujian, Guangdong, Yunnan, Taiwan); India; Micronesia.

Notes: Specimens from the same locality as the holotype have some differences in the aedeagus (Fig. 7) and may be poorly developed. 
Empoasca (Distantasca) faciata (Dworakowska, 1972)

(Figs. 13-24, 159, 172, 185, 198)

Distantasca faciata Dworakowska, 1972: 25.

Empoasca (Distantasca) faciata, Dworakowska, 1976: 4; Dworakowska, 1980: 163; Qin \& Zhang, $2007: 190$.

Specimens examined: China: $2 \precsim 1$, Dinghushan, Guangdong Prov., 17 July 1985, coll. Zhang Yalin; $1 \precsim$, Zhangpu, Fujian Prov., 28 August 2005, 400m, coll. Yang Meixia; 201 o, Diaoluoshan, Hainan Prov., 28 May-2 June 1985, coll. Duan Yani; 2 đ઼, Fanjingshan, Guizhou Prov., 4 July 2001, 950m, coll. Sun Qiang.

Distribution: China (Guangdong, Fujian, Hainan, Yunnan,Guizhou); Vietnam.

Notes: The aedeagal shaft is slightly longer than the preatrium in some specimens.

Empoasca (Distantasca) riora Dworakowska, 1977

(Figs. 25-36, 160, 173, 186, 199)

Empoasca (Distantasca) riora Dworakowska, 1977: 24; Qin \& Zhang, 2007: 190.

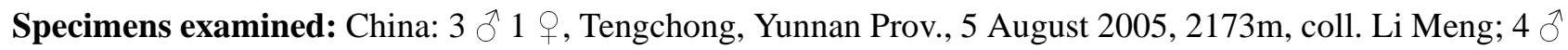
2 ㅇ, Tengchong, Yunnan Prov., 13-14 August 2005, 1930m, coll. Yang Meixia; $1 \stackrel{\jmath}{\jmath}$, Mengyang, Yunnan Prov., 19 July 1999, 840m, coll. Qin Daozheng.

Distribution: China (Yunnan); Vietnam.

Empoasca (Distantasca) tna Dworakowska, 1980

(Figs. 37-48, 161, 174, 187, 200)

Empoasca (Distantasca) tna Dworakowska, 1980: 163.

Specimens examined: China: 2 ภ, Jianfengling, Hainan Prov., 19 May 1983, coll. Zhang Yalin; $2 \precsim$, Guangzhou, Guangdong Prov., 15 July 1985, coll. Zhang Yalin; 1 đૈ, Menglun, Yunnan Prov., 10 April 1982, coll. Zhou Jingruo, Wang Sumei; 1 ग̃, Menglun, Yunnan Prov., 9 December 1999, coll. Dworakowska.

Distribution: China (Hainan, Guangdong, Yunnan); India (West Bengal).

Empoasca (Distantasca) rabava Dworakowska, 1980

(Figs. 49-53)

Empoasca (Distantasca) rabava Dworakowska, 1980: 158.

Distribution: India (Tamil Nadu).

Empoasca (Distantasca) barawa Dworakowska, 1980

(Figs. 54-58)

Empoasca (Distantasca) barawa Dworakowska, 1980: 159.

Distribution: Nepal (Pokhara).

Empoasca (Distantasca) latava Dworakowska, 1981

(Figs. 59-69, 162, 175, 188, 201)

Empoasca (Distantasca) latava Dworakowska, 1981: 159; Qin \& Zhang, 2007: 190. 

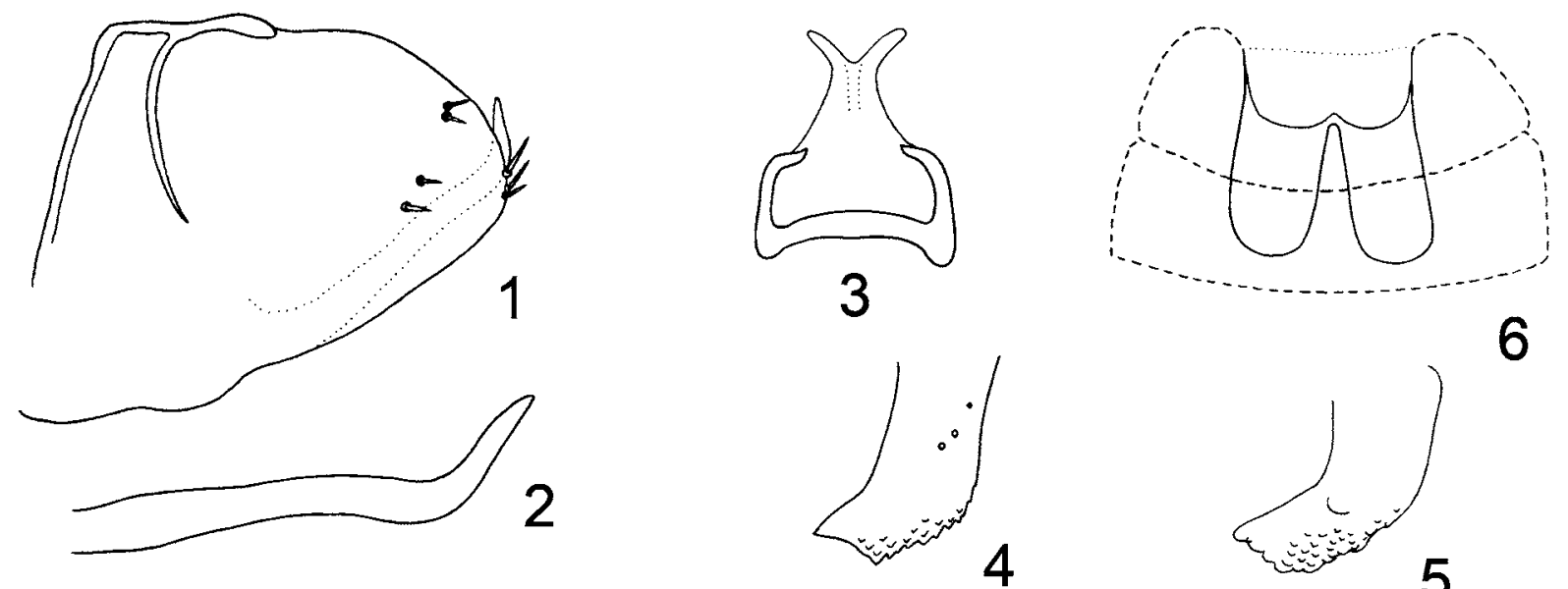

6
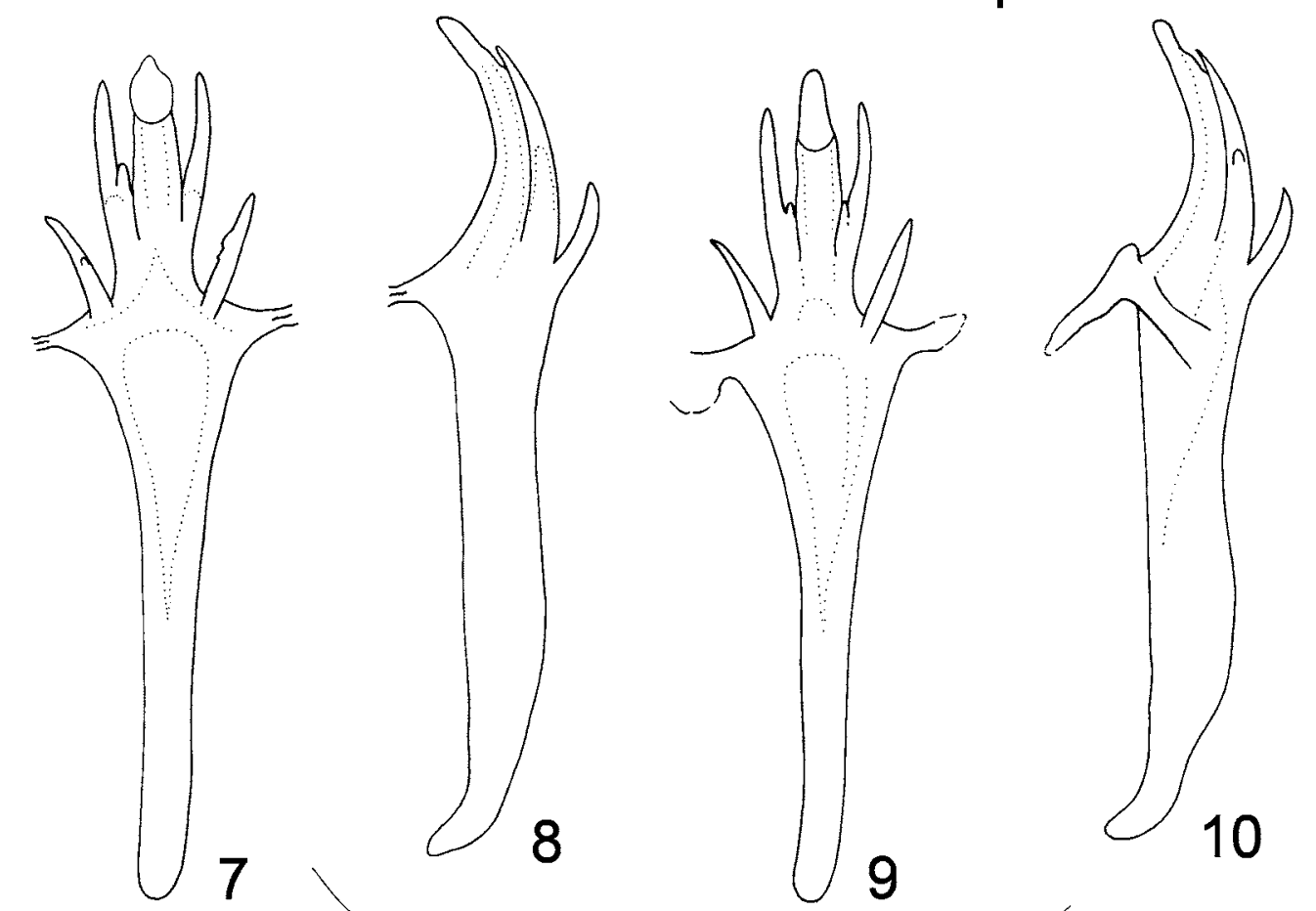

5
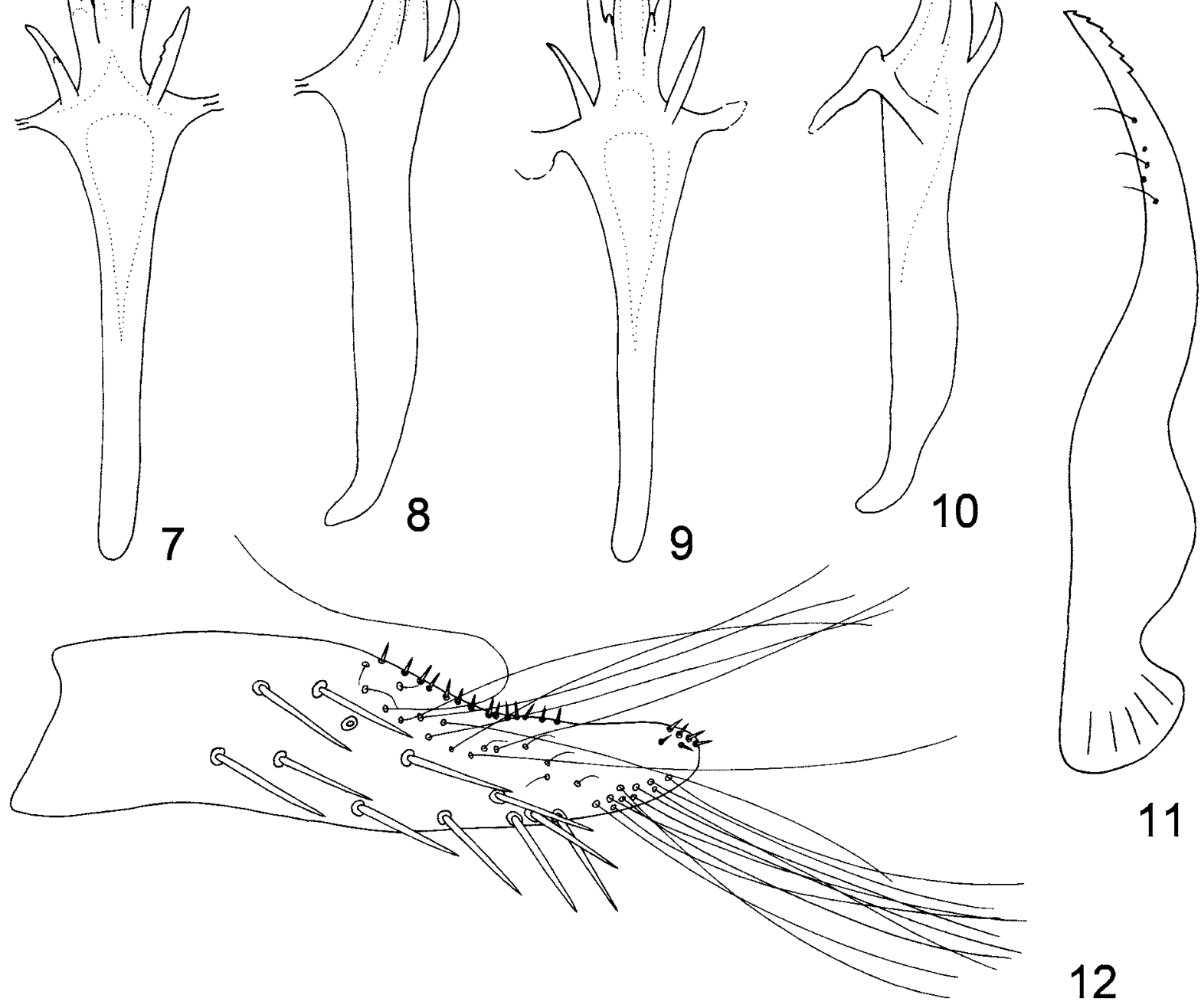

FIGURES 1-12. Empoasca (Distantasca) terminalis Distant, 1, male pygofer, lateral view; 2, ventral pygofer appendage; 3, connective; 4 , anal tube appendage; 5 , anal tube appendage; 6 , abdominal apodemes; 7 , aedeagus, ventral view; 8 , the same, lateral view; 9 , aedeagus, ventral view; 10, the same, lateral view; 11, paramere; 12, subgenital plate (5, 9, 10 after Dworakowska, 1972). 

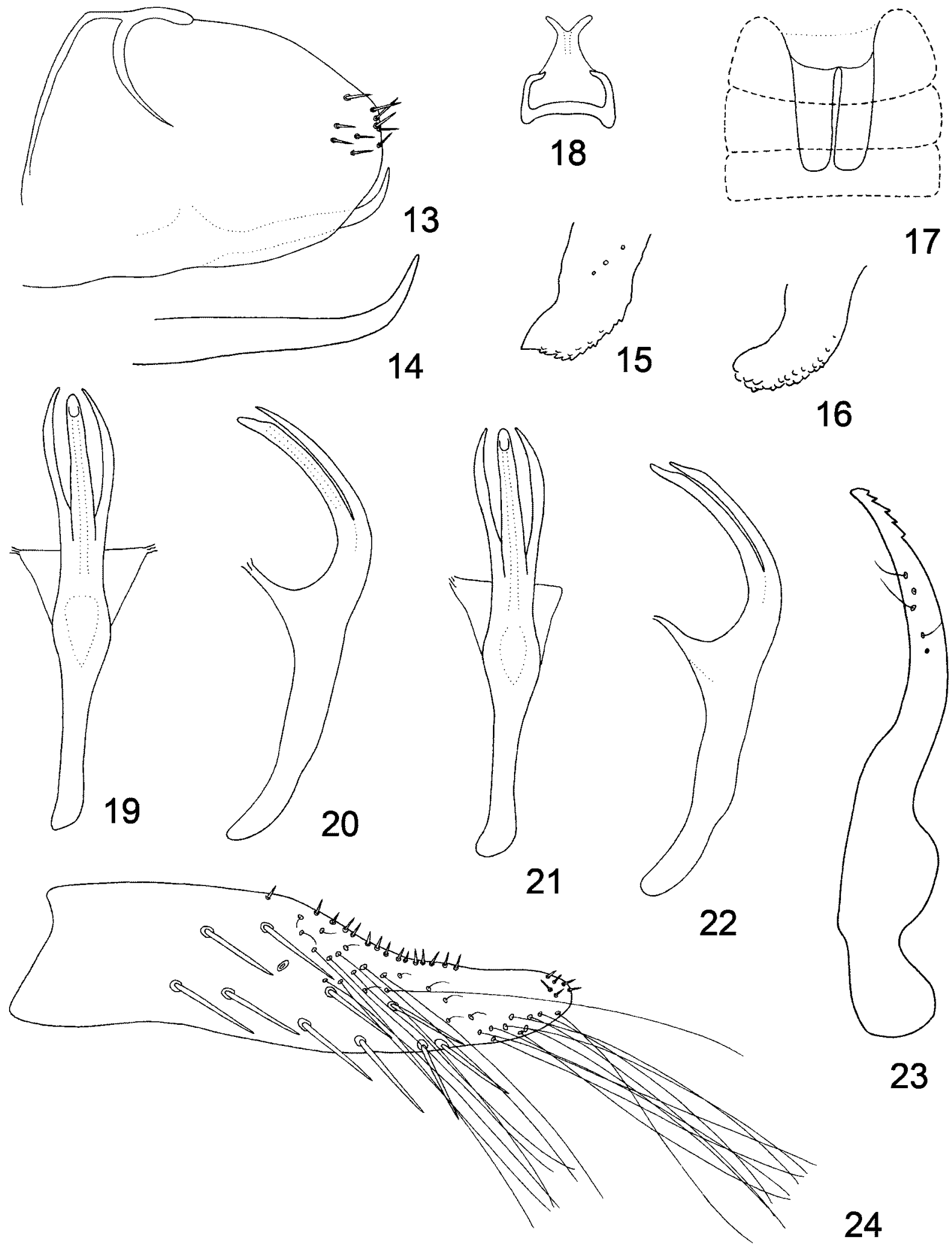

16
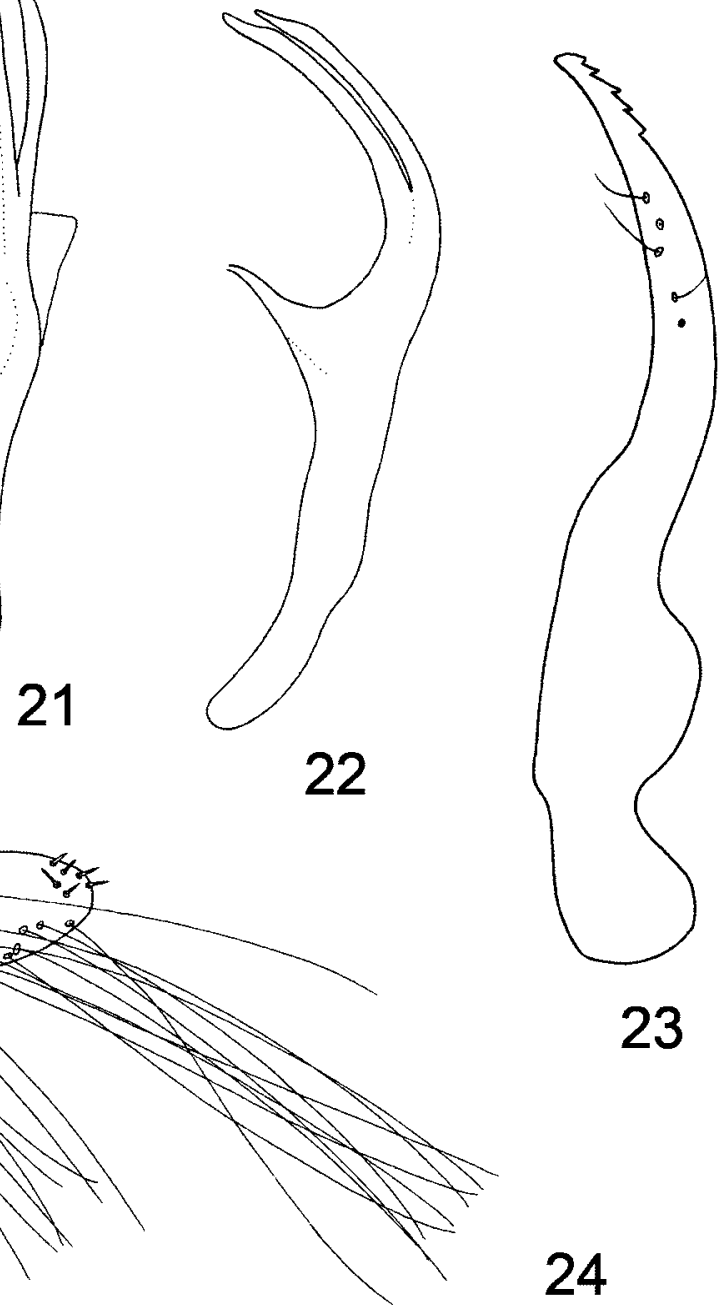

FIGURES 13-24. Empoasca (Distantasca) faciata (Dworakowska), 13, male pygofer, lateral view; 14, ventral pygofer appendage; 15, anal tube appendage; 16, anal tube appendage; 17, abdominal apodemes; 18, connective; 19, aedeagus, ventral view; 20, aedeagus, lateral view; 21, aedeagus, ventral view; 22, aedeagus, lateral view; 23, paramere; 24, subgenital plate (16 after Dworakowska, 1972; 21, 22 after Dworakowska, 1980). 

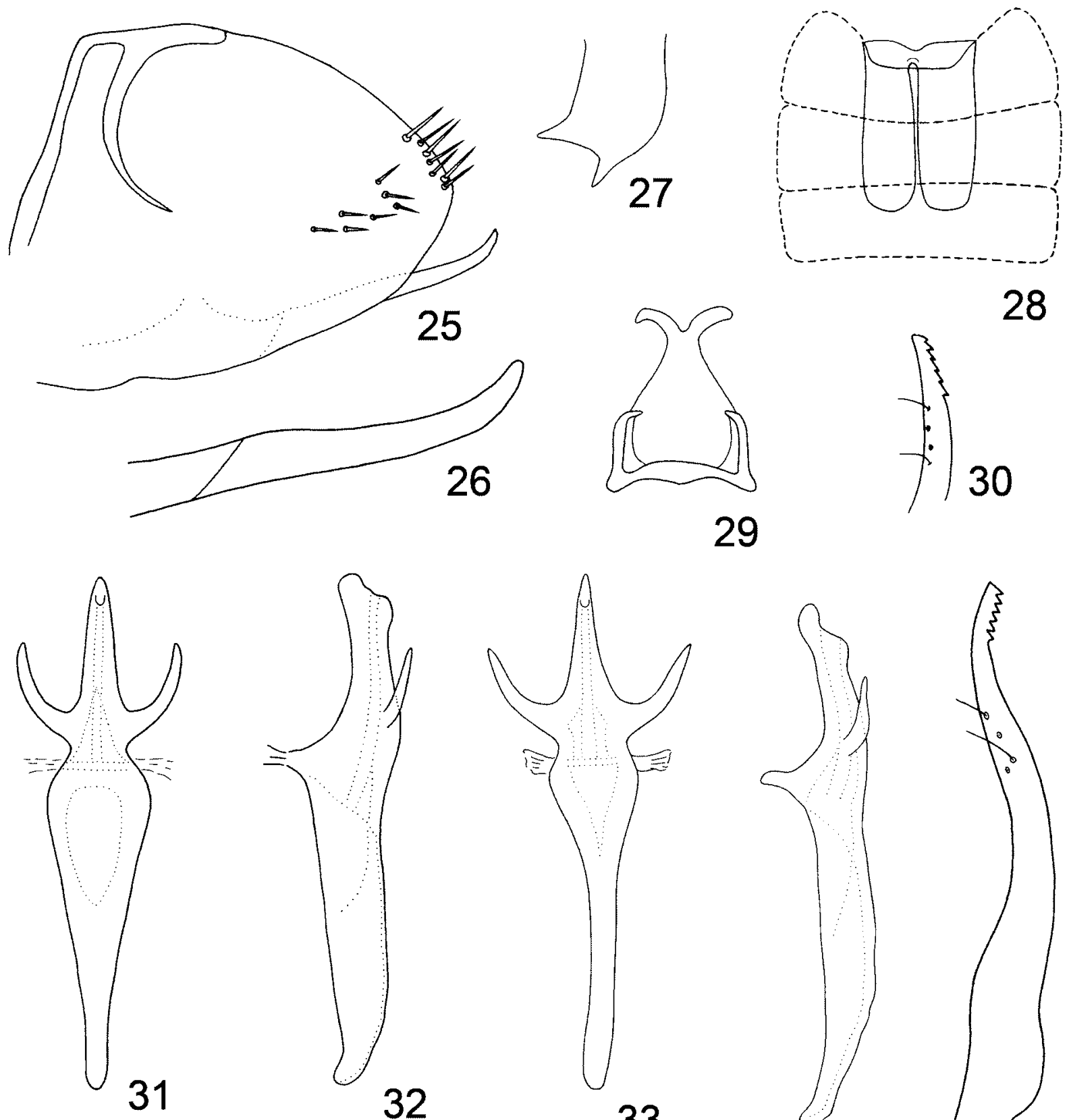

33
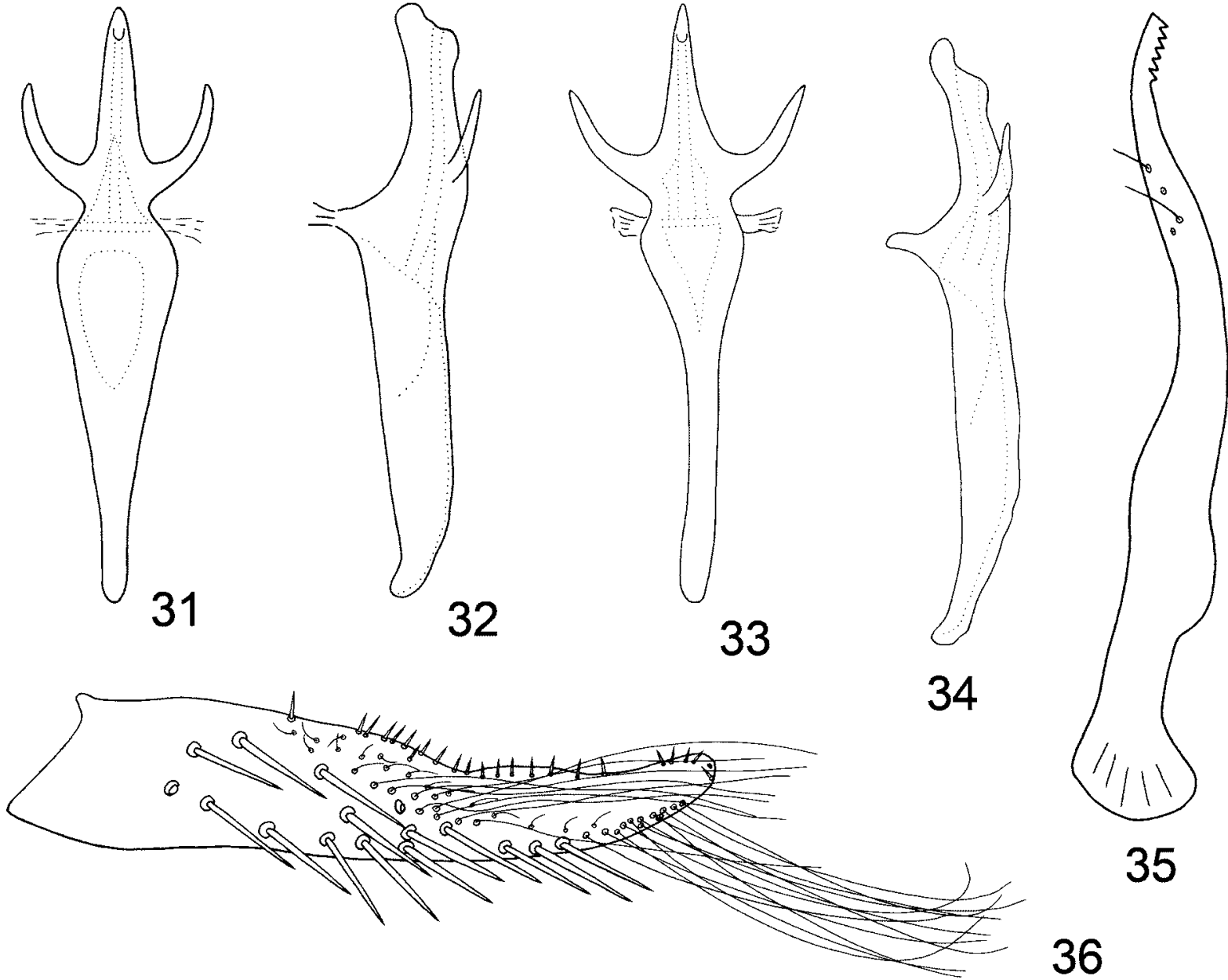

FIGURES 25-36. Empoasca (Distantasca) riora Dworakowska, 25, male pygofer, lateral view; 26, ventral pygofer appendage; 27 , anal tube appendage; 28 , abdominal apodemes; 29 , connective; 30, paramere; 31 , aedeagus, ventral view; 32 , aedeagus, lateral view; 33 , aedeagus, ventral view; 34 , aedeagus, lateral view; 35 , paramere; 36 , subgenital plate ( 30 , 33, 34 after Dworakowska, 1977). 

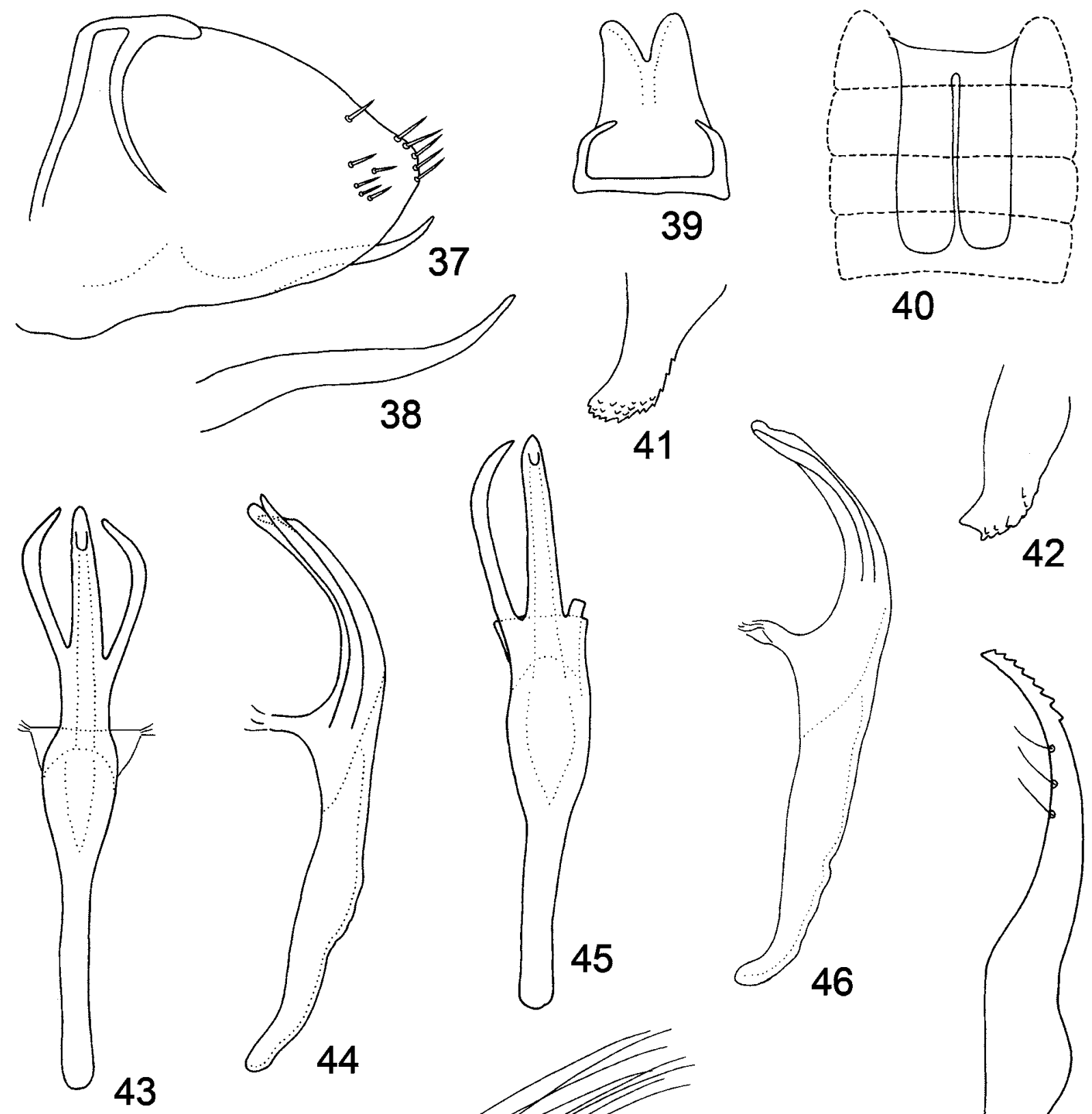

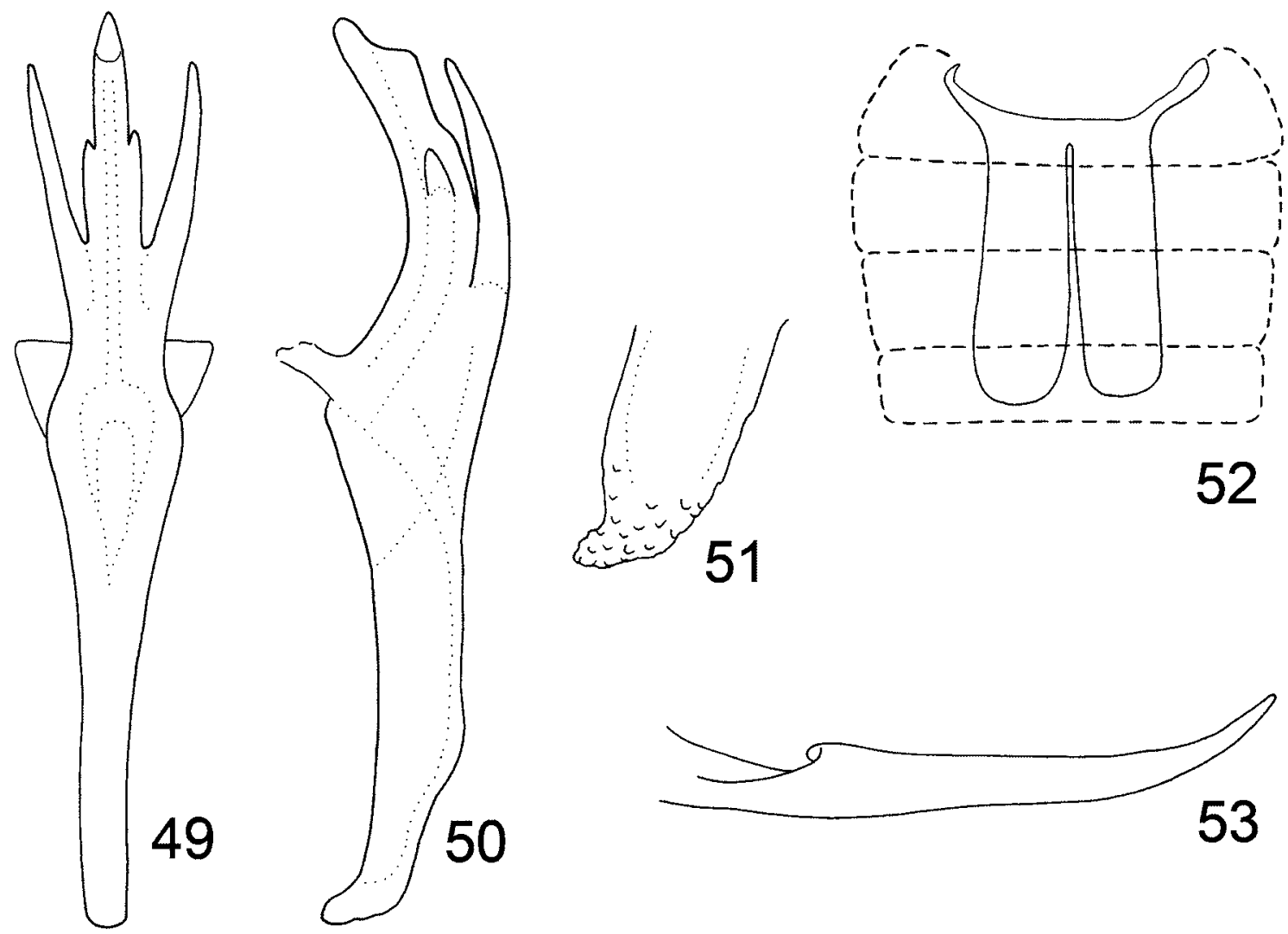

FIGURES 49-53. Empoasca (Distantasca) rabava Dworakowska, 49, aedeagus, ventral view; 50, aedeagus, lateral view; 51, anal tube appendage; 52, abdominal apodemes; 53, ventral pygofer appendage (all after Dworakowska, 1980).

Specimens examined: China: 1 \ 1 , Tengchong, Yunnan Prov., 16 August 2005, 1700m, coll. Li Meng; 2 +, Diaoluoshan, Hainan Prov., 29 May 2007, coll. Duan Yani; 1 đ̆, Yacheng, Hainan Prov., 14 May 1983; 2 đ, Jianfengling, Hainan Prov., 14-18 May 1983; 3 § 2 q, Bawangling, Hainan Prov., 25 May 1983; all were collected by Zhang Yalin.

Distribution: China (Yunnan, Hainan); India.

Notes: Different specimens of this species vary somwhat in the curvature of the basal aedeagal appendages and their degree of fusion to the shaft.

\section{Empoasca (Distantasca) rokasa Dworakowska, 1981}

(Figs. 70-80, 163, 176, 189, 202)

Empoasca (Distantasca) rokasa Dworakowska, 1981: 159; Qin \& Zhang, 2007: 191.

Specimens examined: China: 1 , Shaoshan in Xiangtan, Hunan Prov., 29 July 2002, coll. Sun Qinxia; 1 ภ 1 †, Tengchong, Yunnan Prov., 13-14 August 2005, 1930m, coll. Yang Meixia; 1 đ, Sanchahe in Mengyang, Yunnan Prov., 7 June 1991, 800m, coll. Tian Rungang, Cai Wanzhi and Wang Yinglun; 1 ð, Nangongshan, Yunnan Prov., 17 December 1999, coll. Dworakowska.

Distribution: China (Hunan, Yunnan); Nepal.

Notes: Some specimens vary in having the lateral flanges of the shaft (Fig. 75) broader than in the holotype (Fig. 77). 

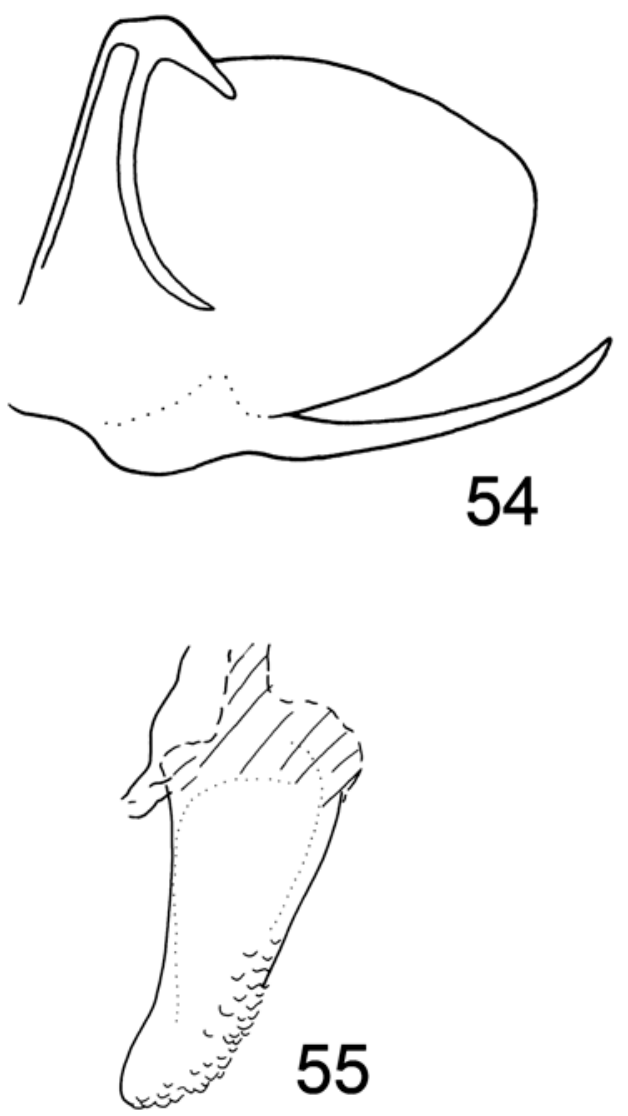
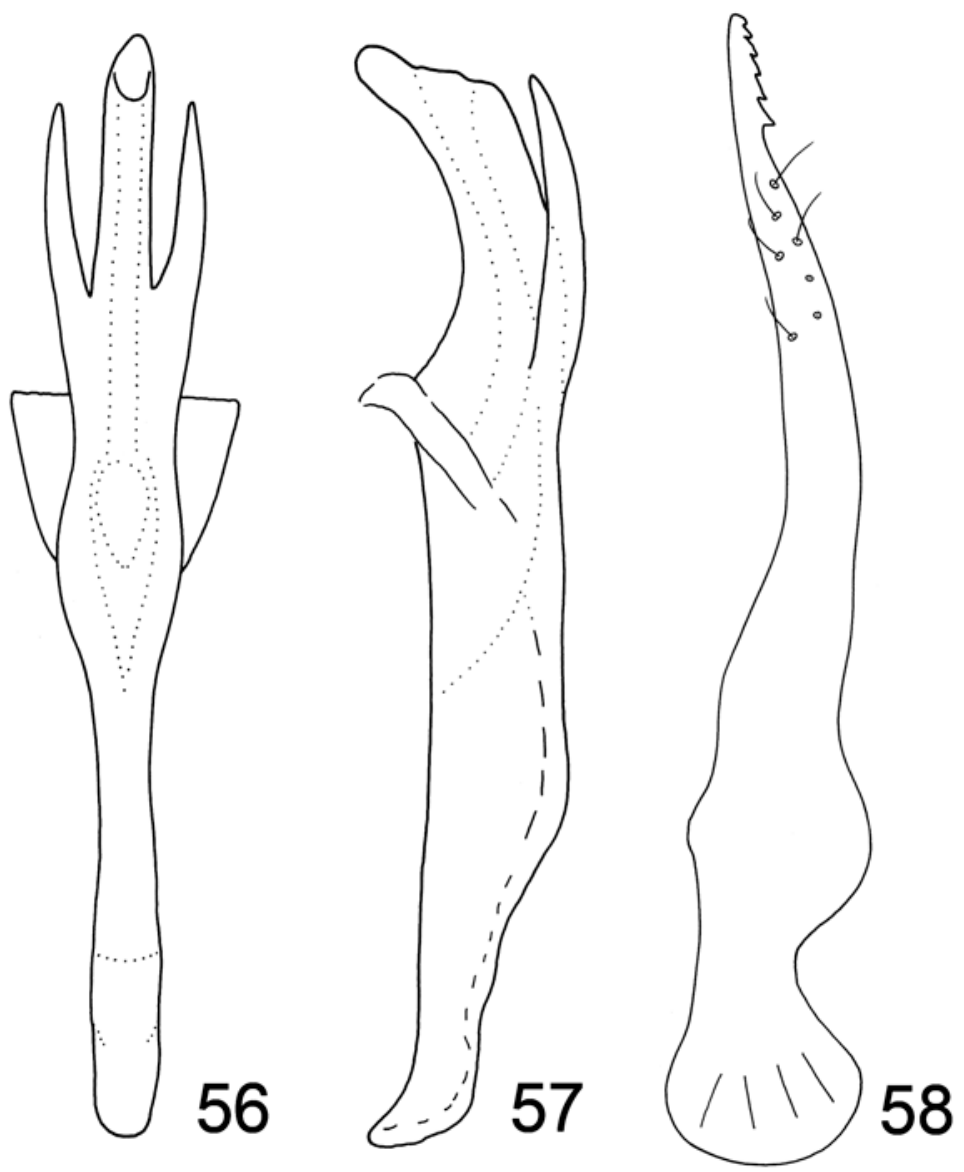

FIGURES 54-58. Empoasca (Distantasca) barawa Dworakowska, 54, male pygofer, lateral view; 55 anal tube appendage; 56, aedeagus, ventral view; 57, aedeagus, lateral view; 58, paramere (all after Dworakowska, 1980).

Empoasca (Distantasca) atika Dworakowska, 1982

(Figs. 81-93, 164, 177, 190, 203)

Empoasca (Distantasca) atika Dworakowska, 1982: 52; Qin \& Zhang, 2007: 191.

Specimens examined: China: $1 \hat{\jmath}$, Hengshan, Hunan Prov., 11 August 1985; 4 3 $\uparrow$, Mangshan, Hunan

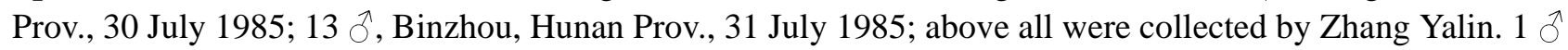
2 , Shaoshan in Xiangtan, Hunan Prov., 29 July 2002, coll. Sun Qinxia.

Distribution: China (Hunan); Japan.

Empoasca (Distantasca) tiaca Dworakowska, 1994

(Figs. 94-105, 165, 178, 191, 204)

Empoasca (Distantasca) tiaca Dworakowska, 1994: 102; Qin \& Zhang, 2007: 191.

Specimens examined: China: 1 đ , Tianpingshan in Sangzhi, Hunan Prov., 14 August 2001, coll. Sun Qiang; 1 đ 1 \&, Tengchong, Yunnan Prov., 5 August 2005, 2173m, coll. Li Meng; 1 đ, Chenzhou, Hunan Prov., 31 July 1985, coll. Zhang Yalin and Chai Yonghui; 7 đ, Fanjingshan, Guizhou Prov., 28 July 2001, 600m, coll. Sun Qiang; 1 đ̃, Tengchong, Yunnan Prov., 20 November 1999, 1600m, coll. Qin Daozheng. 
Distribution: China (Yunnan, Guizhou, Hunan); Sikkim.

Notes: In some specimens, the anal tube appendage is broader than that of the holytype.

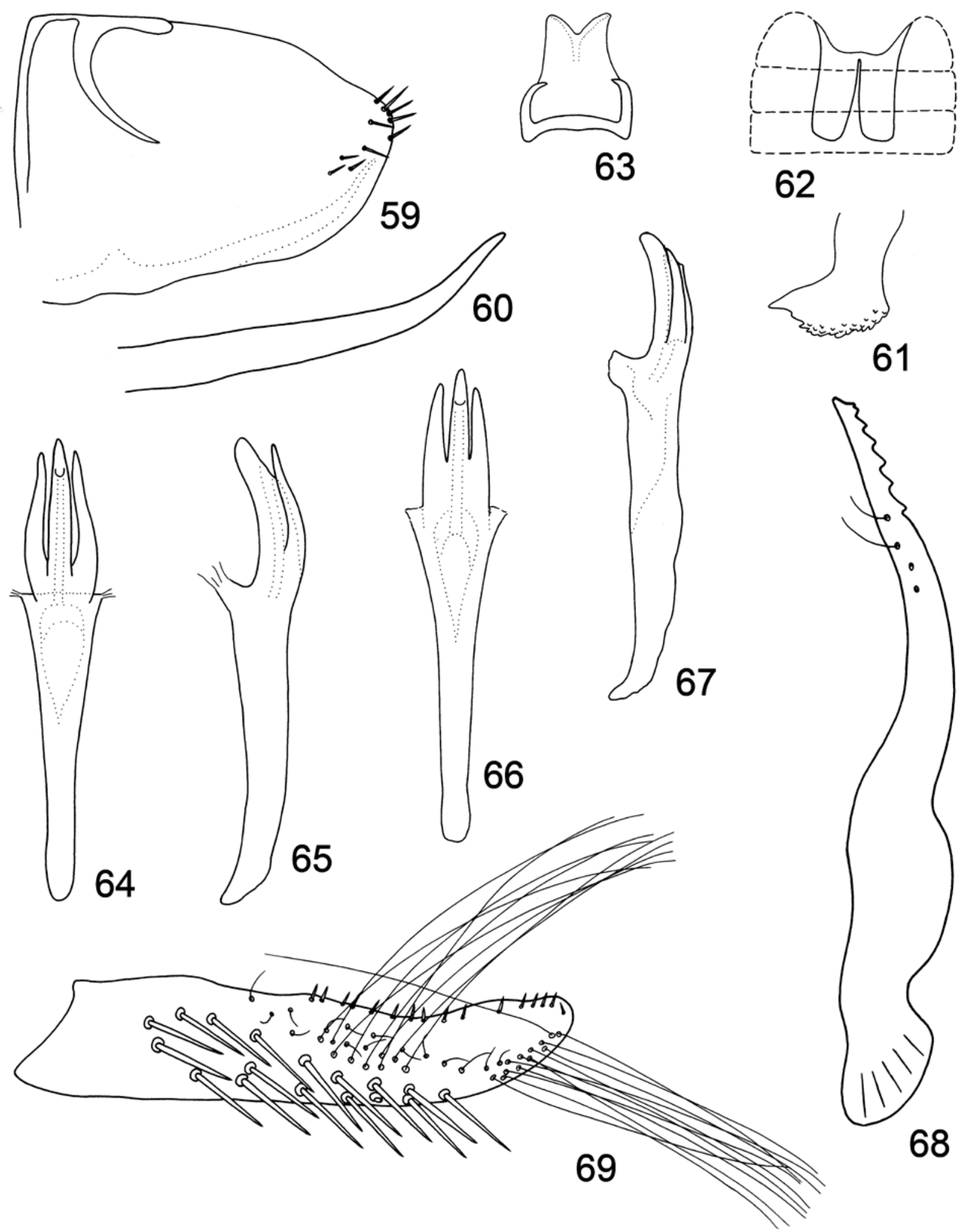

FIGURES 59-69. Empoasca (Distantasca) latava Dworakowska, 59, male pygofer, lateral view; 60, ventral pygofer appendage; 61 , anal tube appendage; 62, abdominal apodemes; 63, connective; 64, aedeagus, ventral view; 65, aedeagus, lateral view; 66, aedeagus, ventral view; 67, aedeagus, lateral view; 68 , paramere; 69 , subgenital plate (66, 67 after Dworakowska, 1981). 

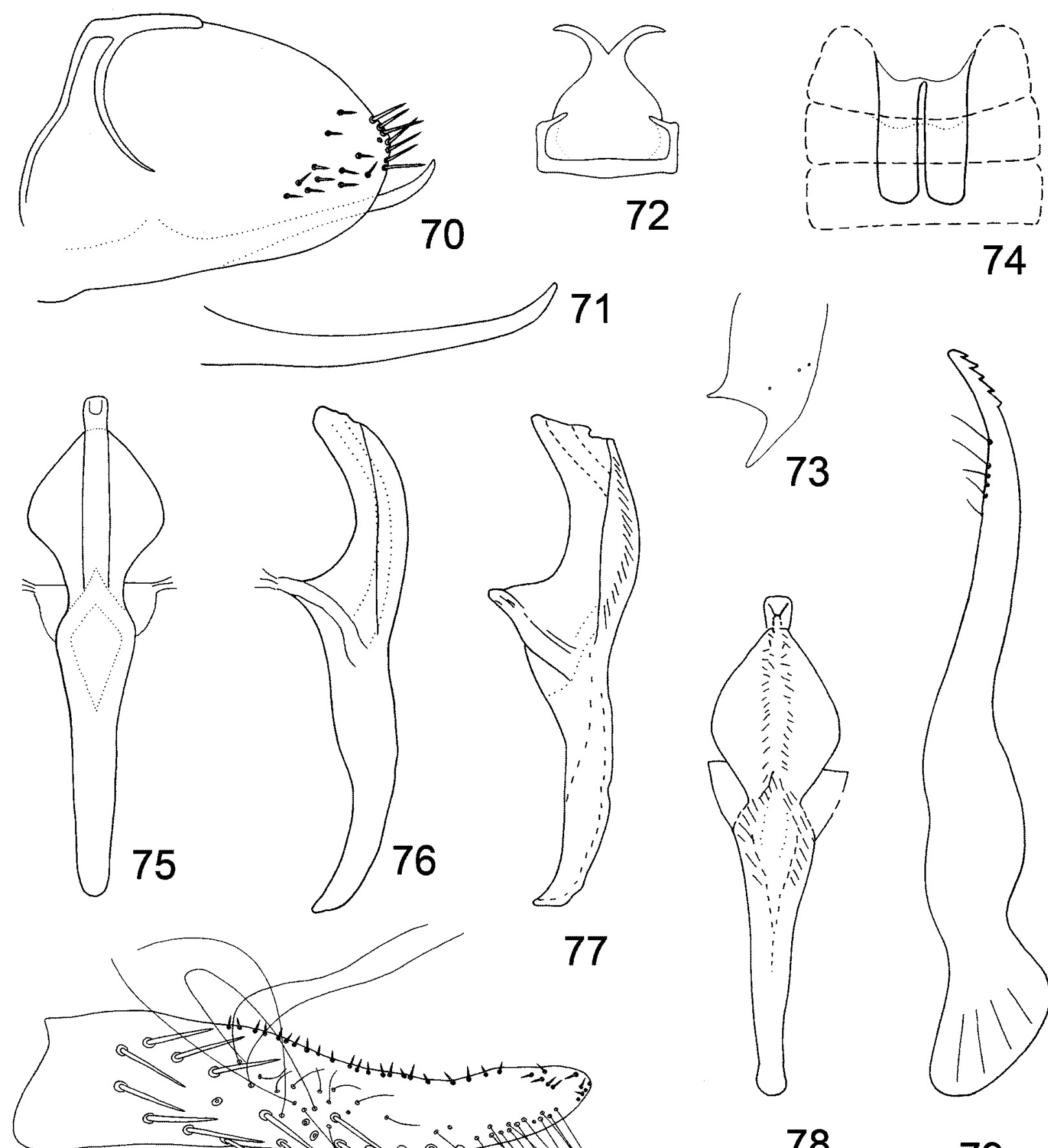

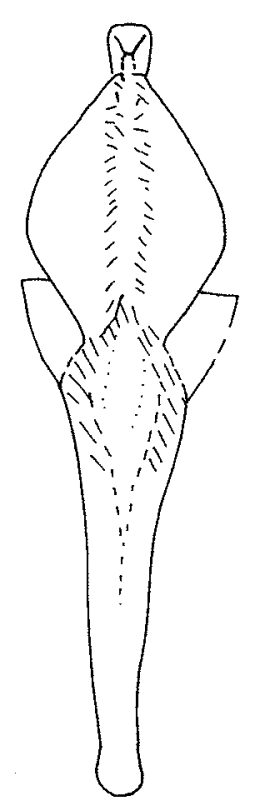

78

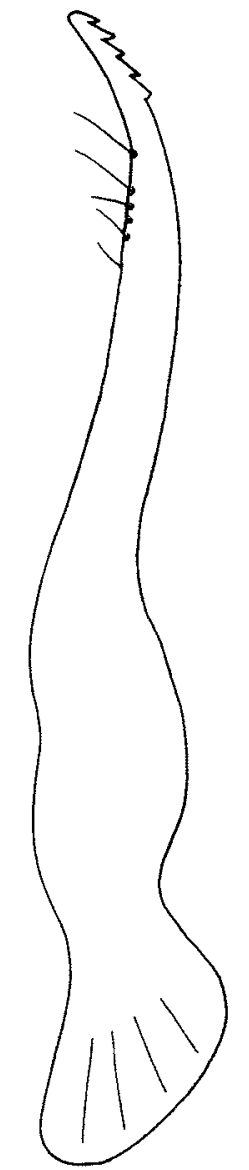

79

FIGURES 70-80. Empoasca (Distantasca) rokasa Dworakowska, 70, male pygofer, lateral view; 71, ventral pygofer appendage; 72, connective; 73, anal tube appendage; 74, abdominal apodemes; 75, aedeagus, ventral view; 76, aedeagus, lateral view; 77, aedeagus, ventral view; 78, aedeagus, lateral view; 79, paramere; 80, subgenital plate. (77, 78 after Dworakowska, 1981) 

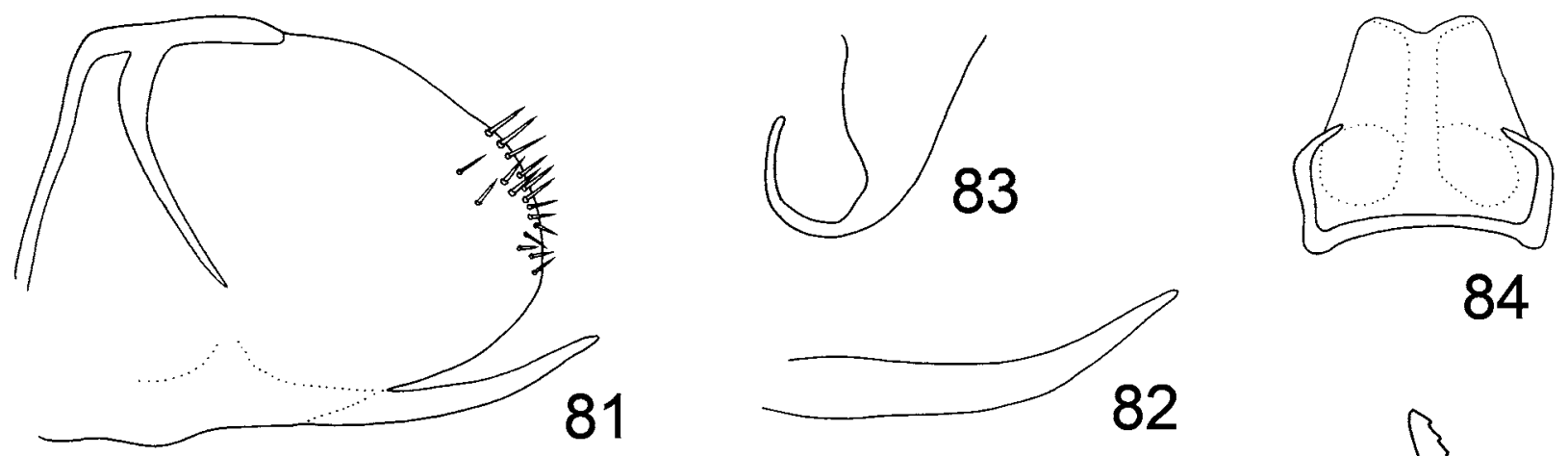

84
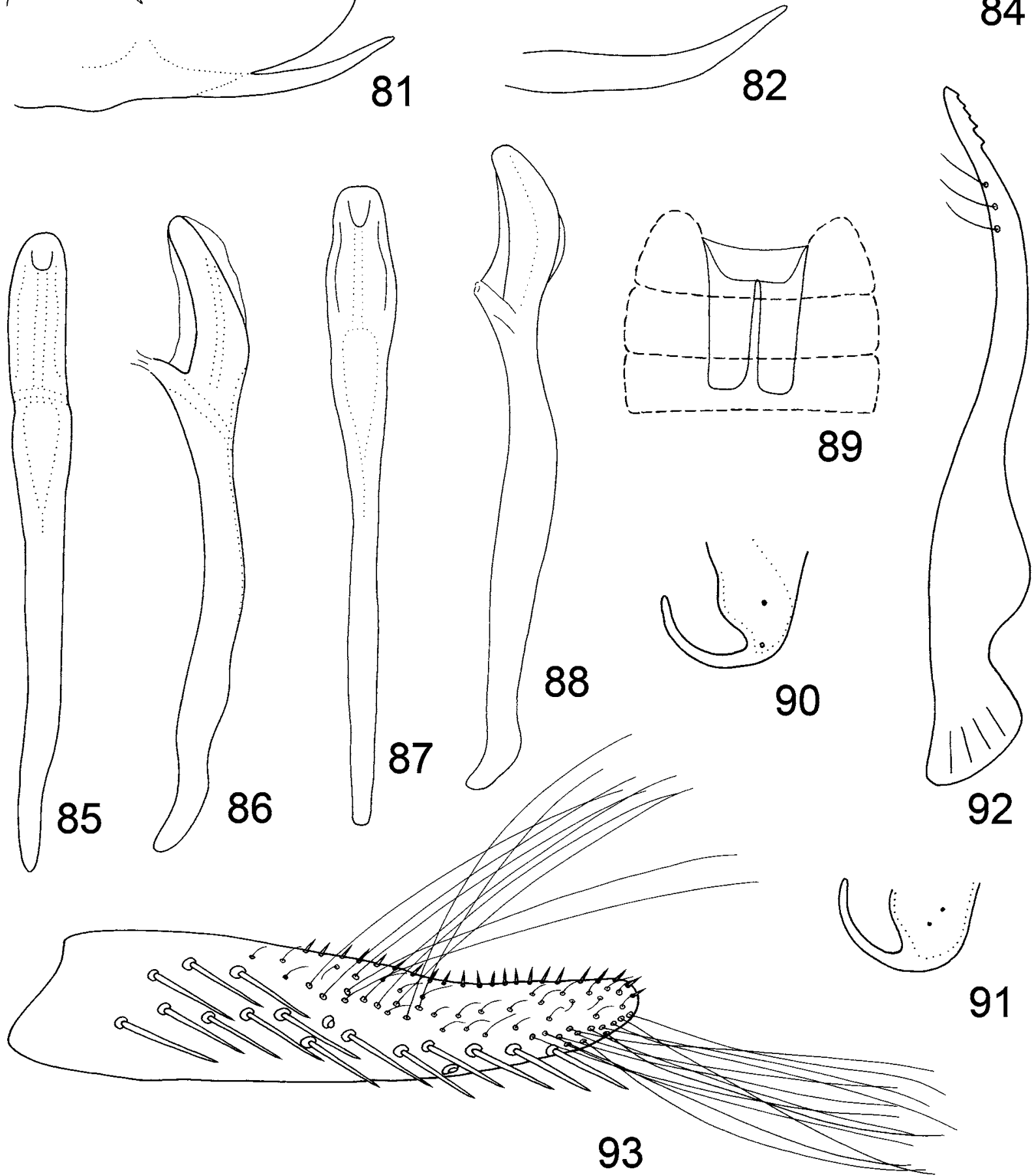

FIGURES 81-93. Empoasca (Distantasca) atika Dworakowska, 81, male pygofer, lateral view; 82, ventral pygofer appendage; 83, anal tube appendage; 84 , connective; 85 , aedeagus, ventral view; 86, aedeagus, lateral view; 87 , aedeagus, ventral view; 88 , aedeagus, lateral view; 89 , abdominal apodemes; 90 , anal tube appendage; 91 , anal tube appendage; 92, paramere; 93, subgenital plate. $(87,88,90,91$ after Dworakowska, 1982) 

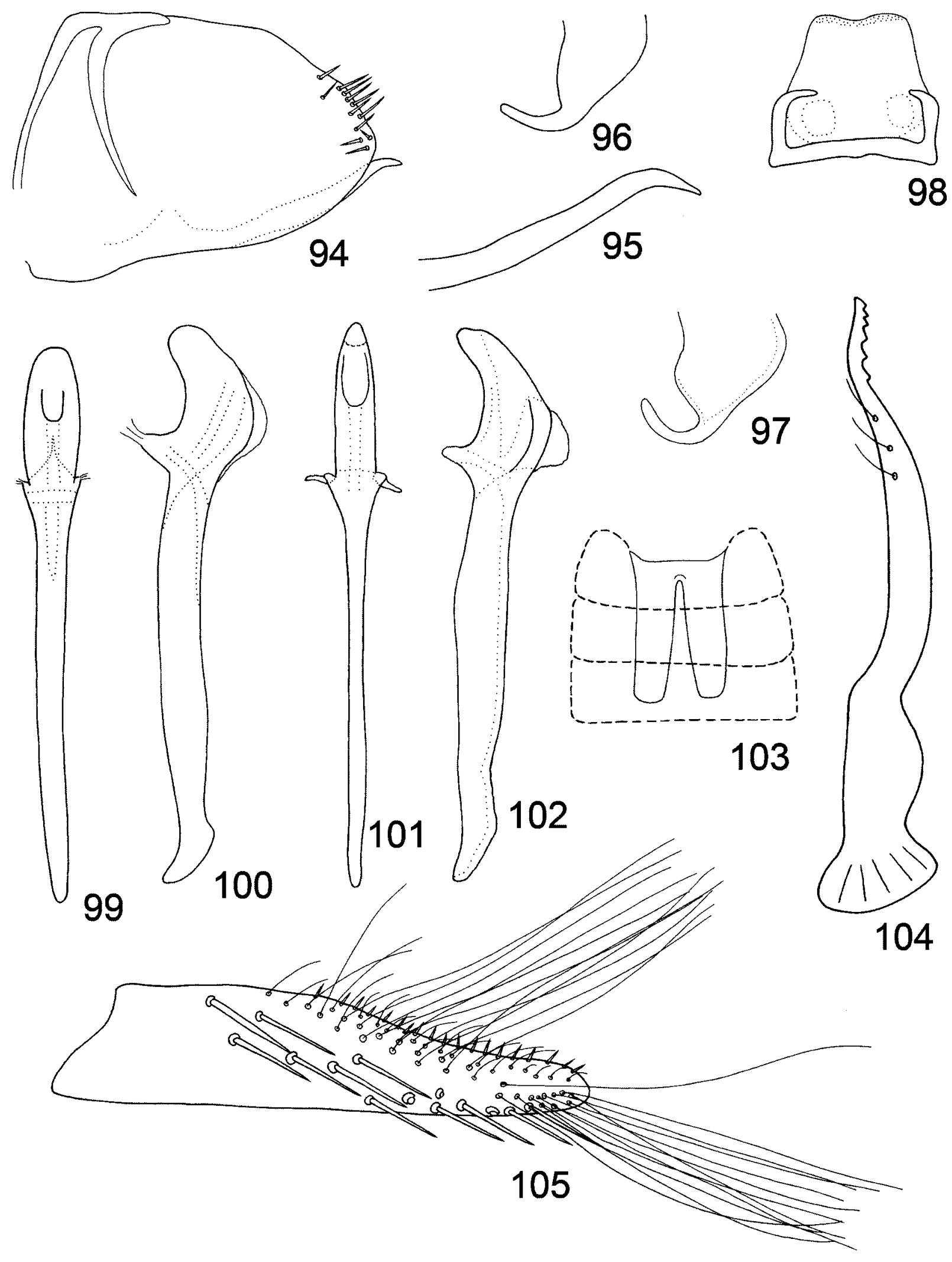

FIGURES 94-105. Empoasca (Distantasca) tiaca Dworakowska, 94, male pygofer, lateral view; 95, ventral pygofer appendage; 96, anal tube appendage; 97, anal tube appendage; 98, connective; 99, aedeagus, ventral view; 100, aedeagus, lateral view; 101, aedeagus, ventral view; 102, aedeagus, lateral view; 103, abdominal apodemes; 104, paramere; 105, subgenital plate. (97, 101, 102 after Dworakowska, 1994) 

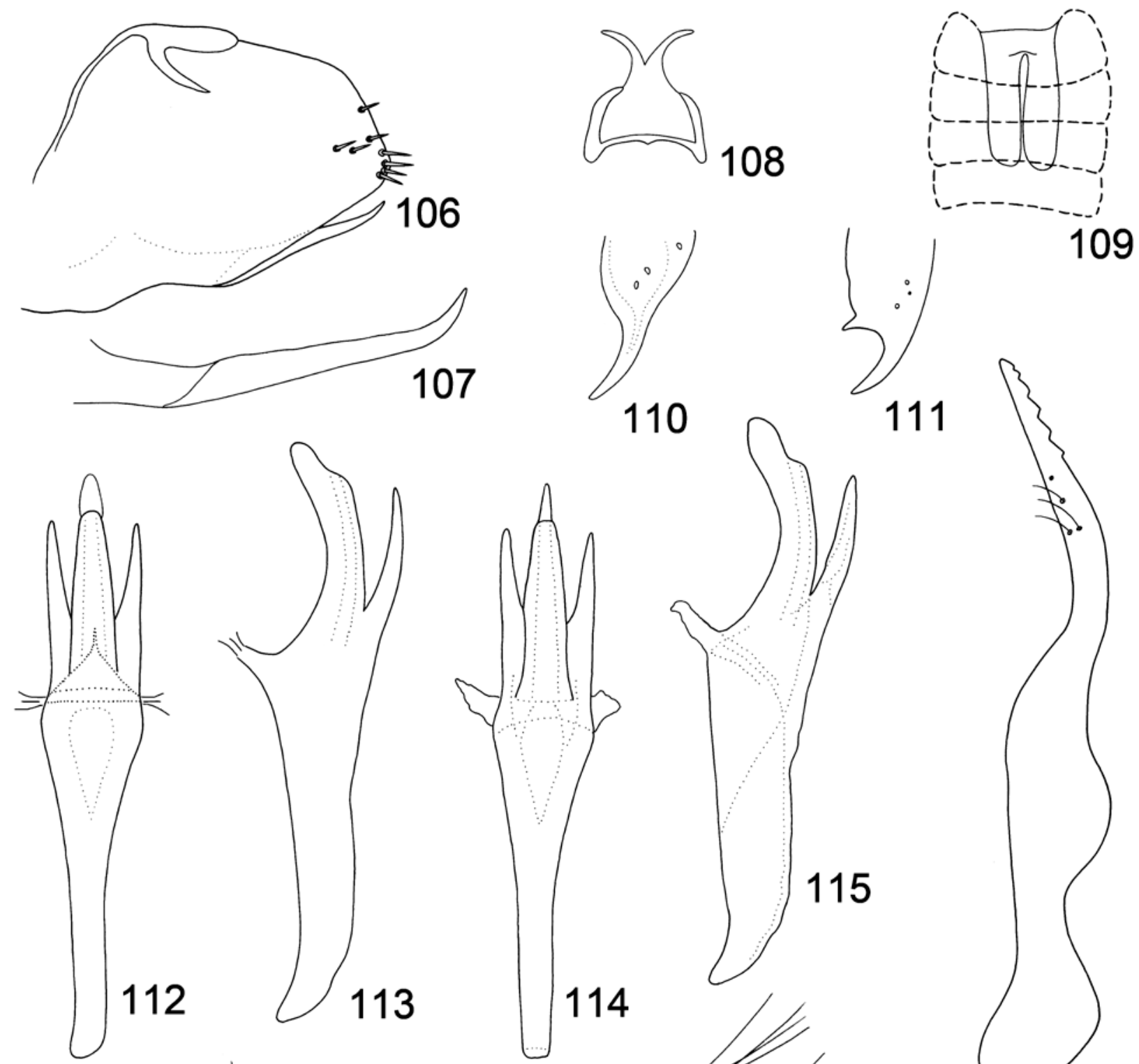

110
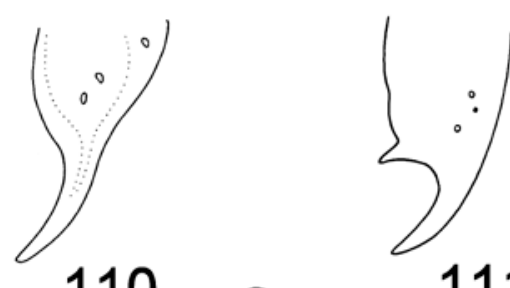

109

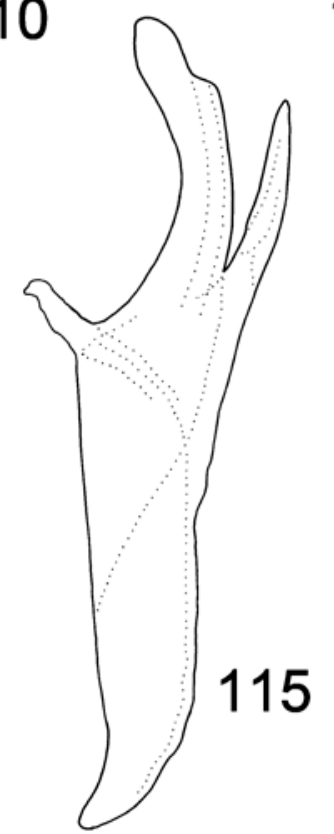

111
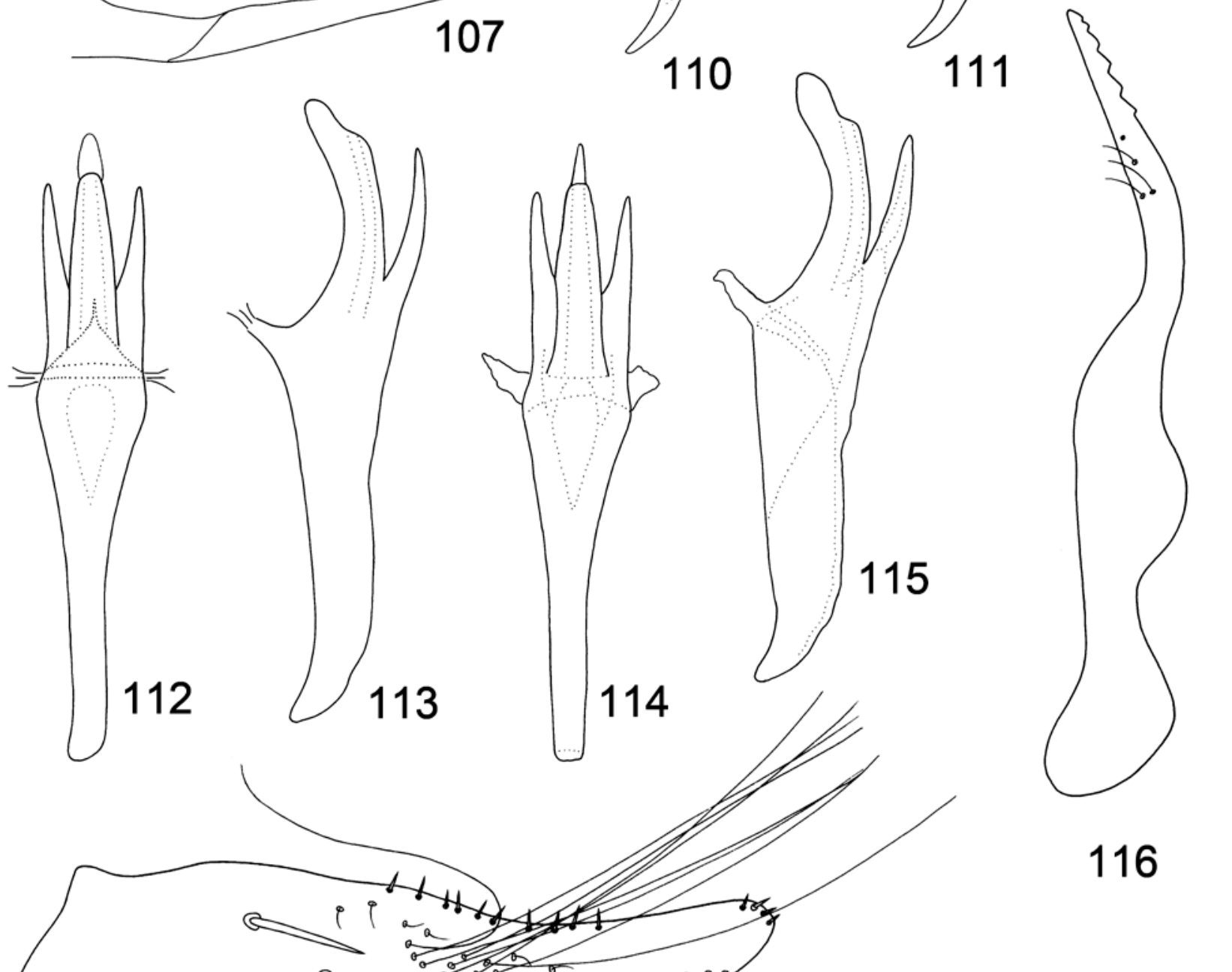

116

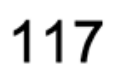

FIGURES 106-117. Empoasca (Distantasca) bulbosa Dworakowska, 106, male pygofer, lateral view; 107, ventral pygofer appendage; 108, connective; 109, abdominal apodemes; 110, anal tube appendage; 111, anal tube appendage; 112, aedeagus, ventral view; 113, the same, lateral view; 114, aedeagus, ventral view; 115, the same, lateral view; 116, paramere; 117, subgenital plate. (110, 114, 115 after Dworakowska, 1994) 


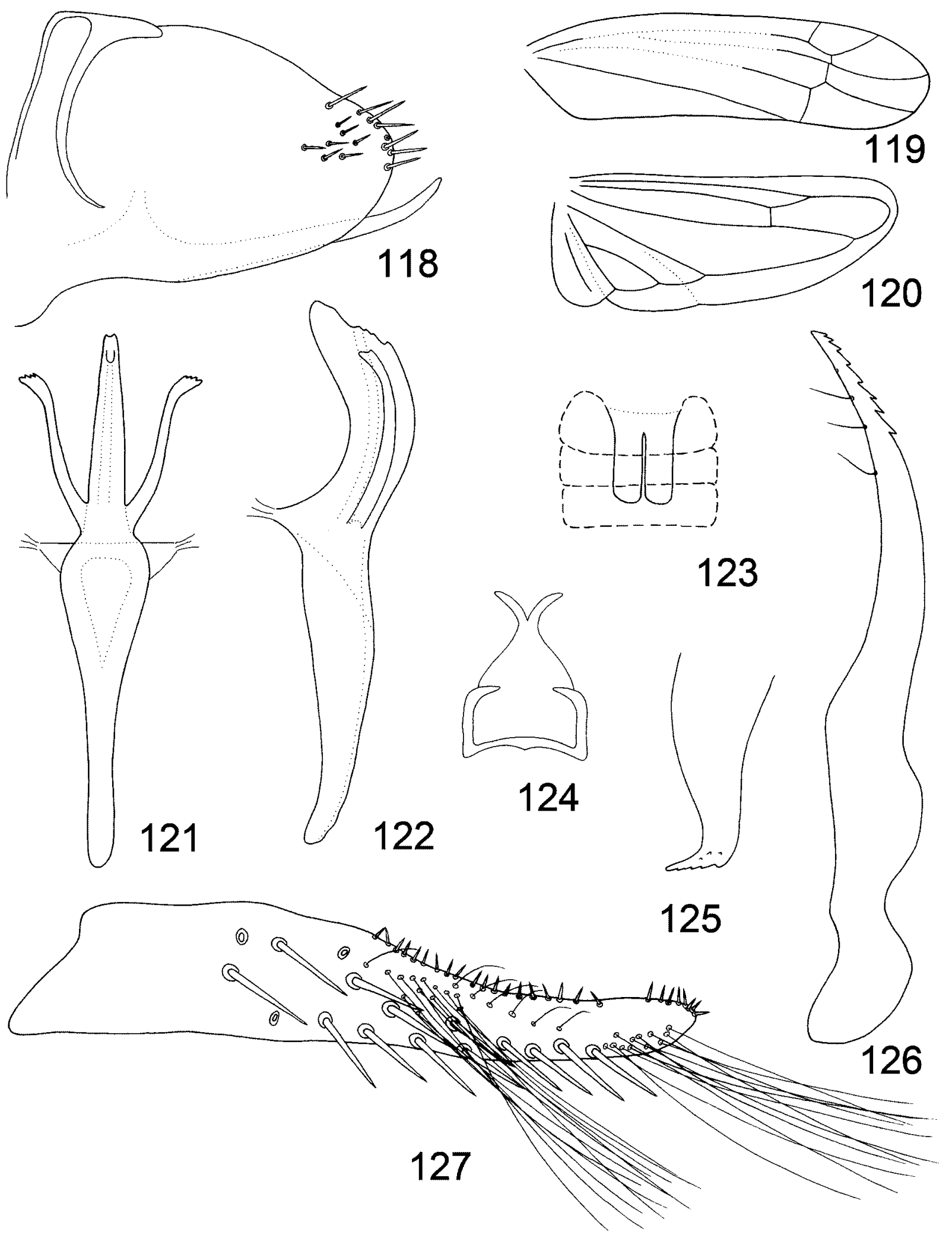

FIGURES 118-127. Empoasca (Distantasca) tuberculata Zhang \& Liu n. sp., 118, male pygofer, lateral view; 119, forewing; 120, hindwing; 121, aedeagus, ventral view; 122, the same, lateral view; 123, abdominal apodemes; 124, connective; 125, anal tube appendage; 126, paramere; 127, subgenital plate. 

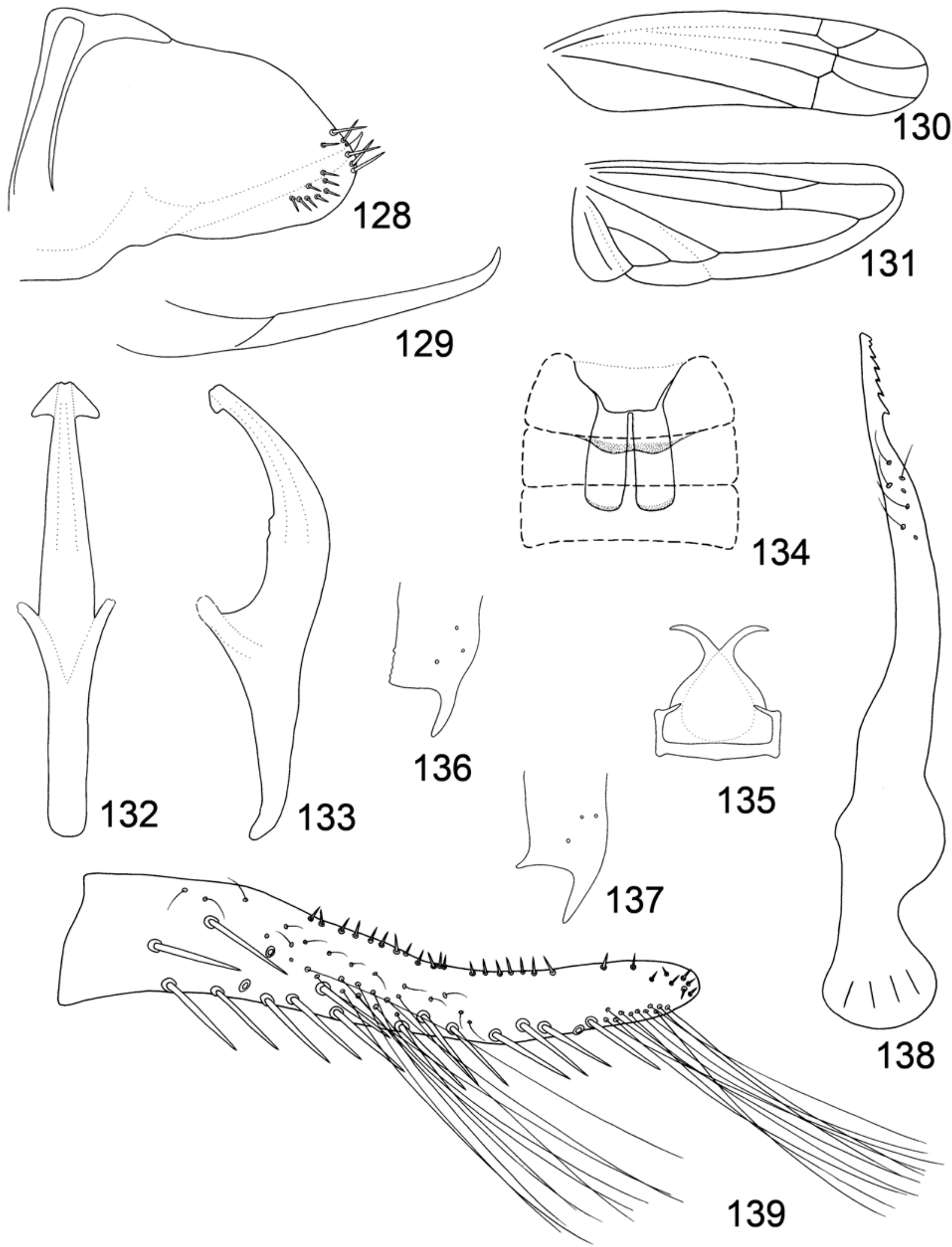

FIGURES 128-139. Empoasca (Distantasca) latibasis Zhang \& Liu n. sp., 128, male pygofer, lateral view; 129, ventral pygofer appendage; 130, forewing; 131, hindwing; 132, aedeagus, ventral view; 133, the same, lateral view; 134, abdominal apodemes; 135, connective; 136, anal tube appendage; 137, anal tube appendage; 138, paramere; 139, subgenital plate. 


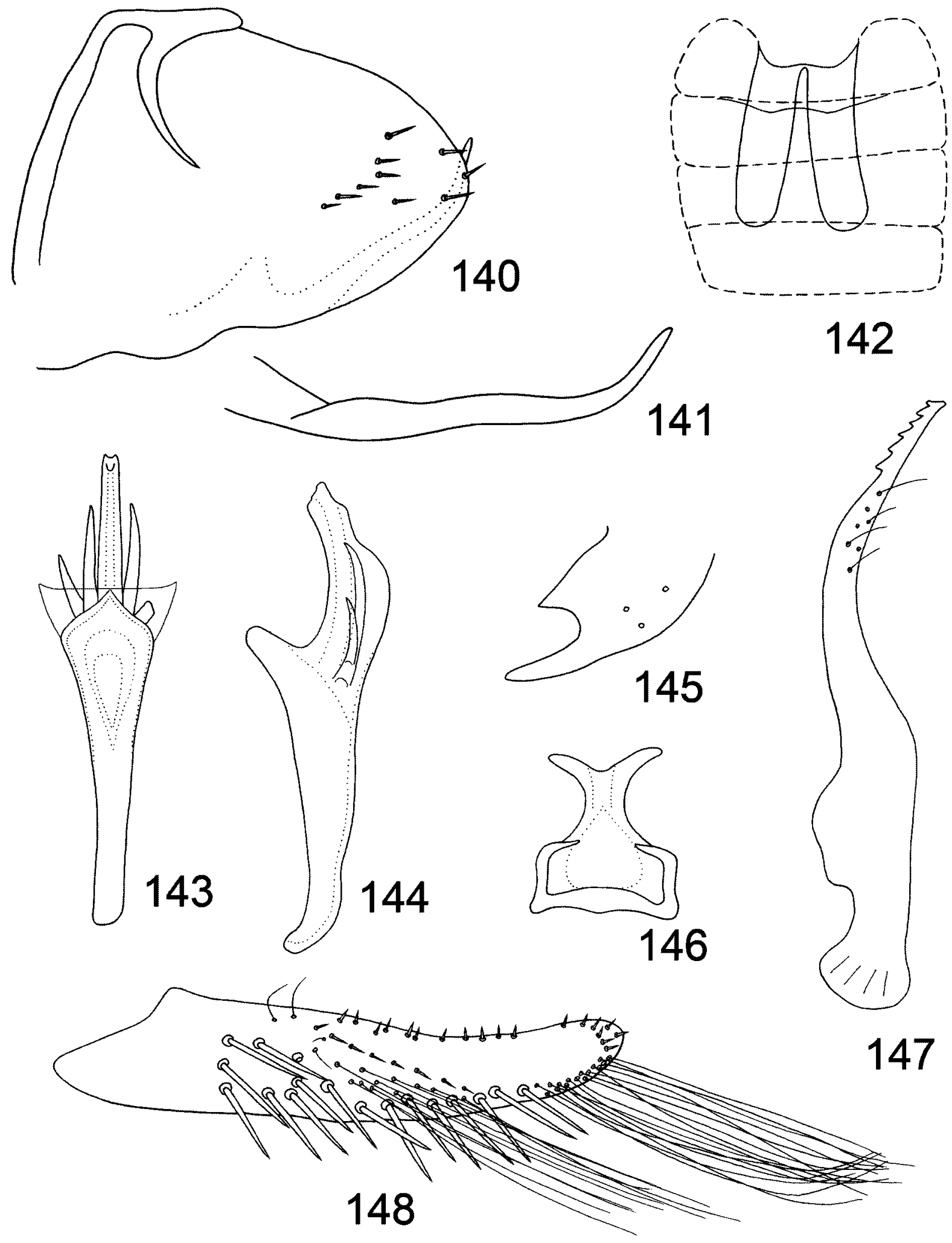

FIGURES 140-148. Empoasca (Distantasca) paraterminalis Qin \& Zhang, 140, male pygofer, lateral view; 141, ventral pygofer appendage; 142, abdominal apodemes; 143, aedeagus, ventral view; 144, the same, lateral view; 145 , anal tube appendage; 146, connective; 147, paramere; 148, subgenital plate. 


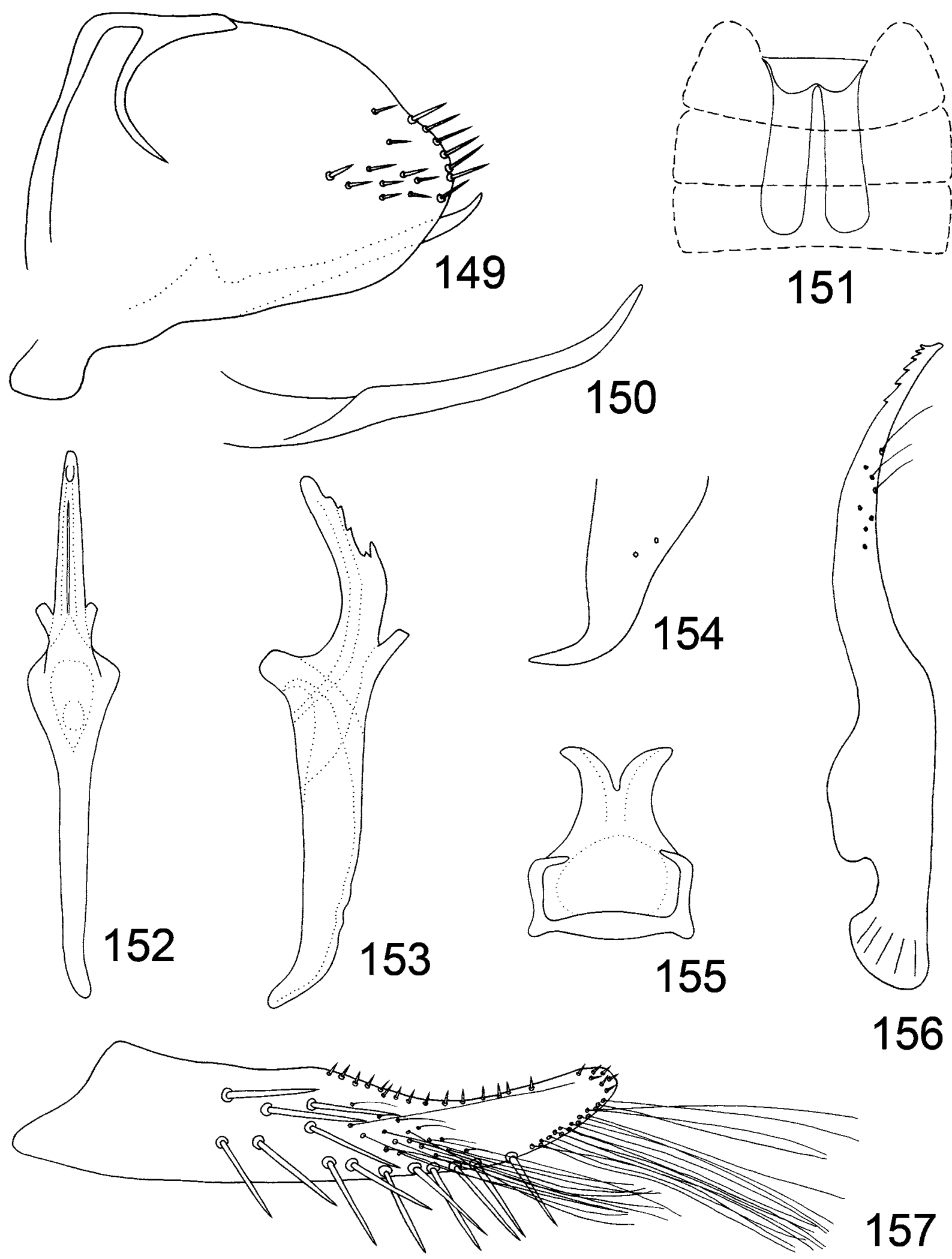

FIGURES 149-157. Empoasca (Distantasca) serratipenis Qin \& Zhang, 149, male pygofer, lateral view; 150, ventral pygofer appendage; 151, abdominal apodemes; 152, aedeagus, ventral view; 153, the same, lateral view; 154, anal tube appendage; 155, connective; 156, paramere; 157, subgenital plate. 

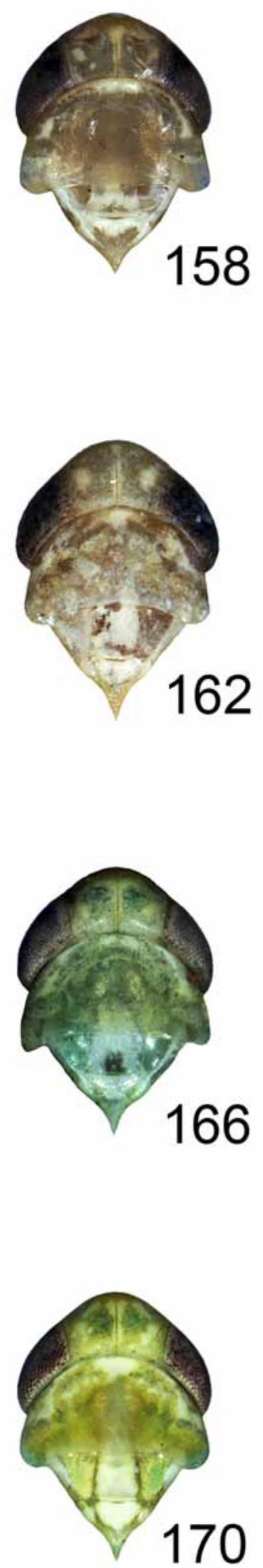
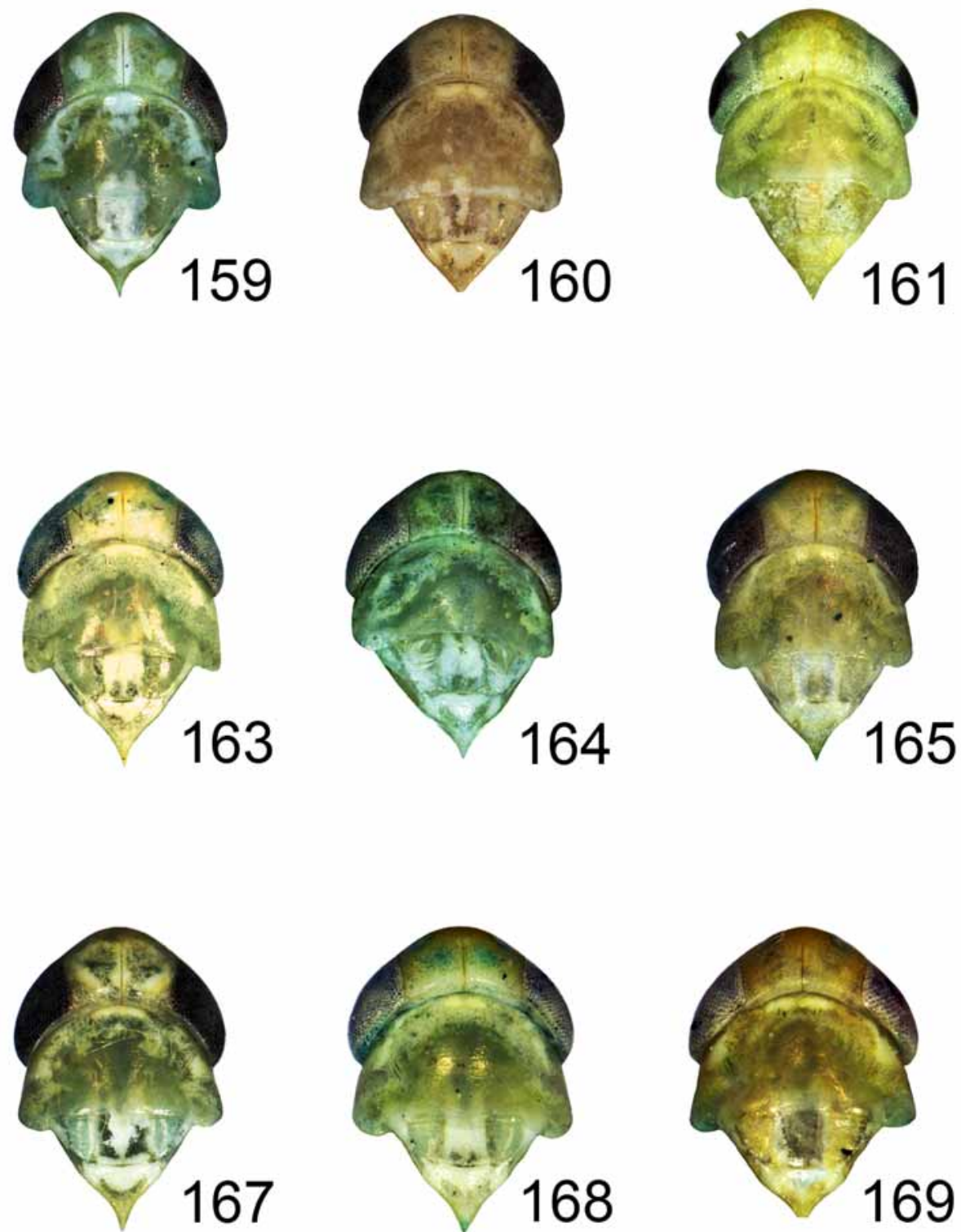

FIGURES 158-170. Heads and thoraxes, dorsal view. 158, Empoasca (Distantasca) terminalis Distant, 1918; 159, Empoasca (Distantasca) faciata (Dworakowska, 1972); 160, Empoasca (Distantasca) riora Dworakowska, 1977; 161, Empoasca (Distantasca) tna Dworakowska, 1980; 162, Empoasca (Distantasca) latava Dworakowska, 1981; 163, Empoasca (Distantasca) rokasa Dworakowska, 1981; 164, Empoasca (Distantasca) atika Dworakowska, 1982; 165, Empoasca (Distantasca) tiaca Dworakowska, 1994; 166, Empoasca (Distantasca) bulbosa Dworakowska, 1994; 167, Empoasca (Distantasca) paraterminalis Qin \& Zhang; 168, Empoasca (Distantasca) serratipenis Qin \& Zhang; 169, Empoasca (Distantasca) tuberculata Zhang \& Liu sp. n.; 170, Empoasca (Distantasca) latibasis Zhang \& Liu sp. n. 

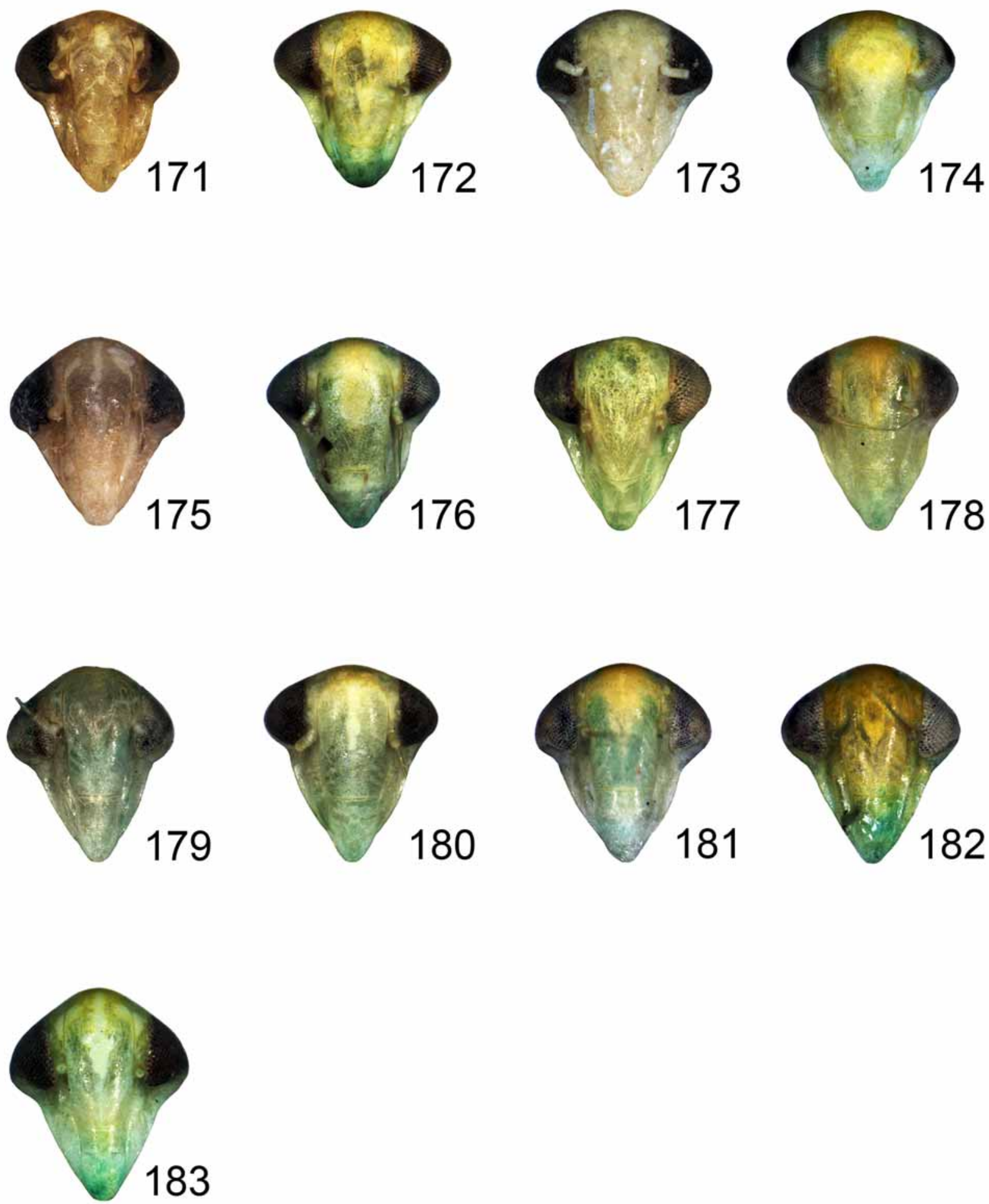

FIGURES 171-183. Faces. 171, Empoasca (Distantasca) terminalis Distant; 172, Empoasca (Distantasca) faciata (Dworakowska); 173, Empoasca (Distantasca) riora Dworakowska; 174, Empoasca (Distantasca) tna Dworakowska; 175, Empoasca (Distantasca) latava Dworakowska; 176, Empoasca (Distantasca) rokasa Dworakowska; 177, Empoasca (Distantasca) atika Dworakowska; 178, Empoasca (Distantasca) tiaca Dworakowska; 179, Empoasca (Distantasca) bulbosa Dworakowska; 180, Empoasca (Distantasca) paraterminalis Qin \& Zhang; 181, Empoasca (Distantasca) serratipenis Qin \& Zhang; 182, Empoasca (Distantasca) tuberculata Zhang \& Liu sp. n.; 183, Empoasca (Distantasca) latibasis Zhang \& Liu sp. n. 

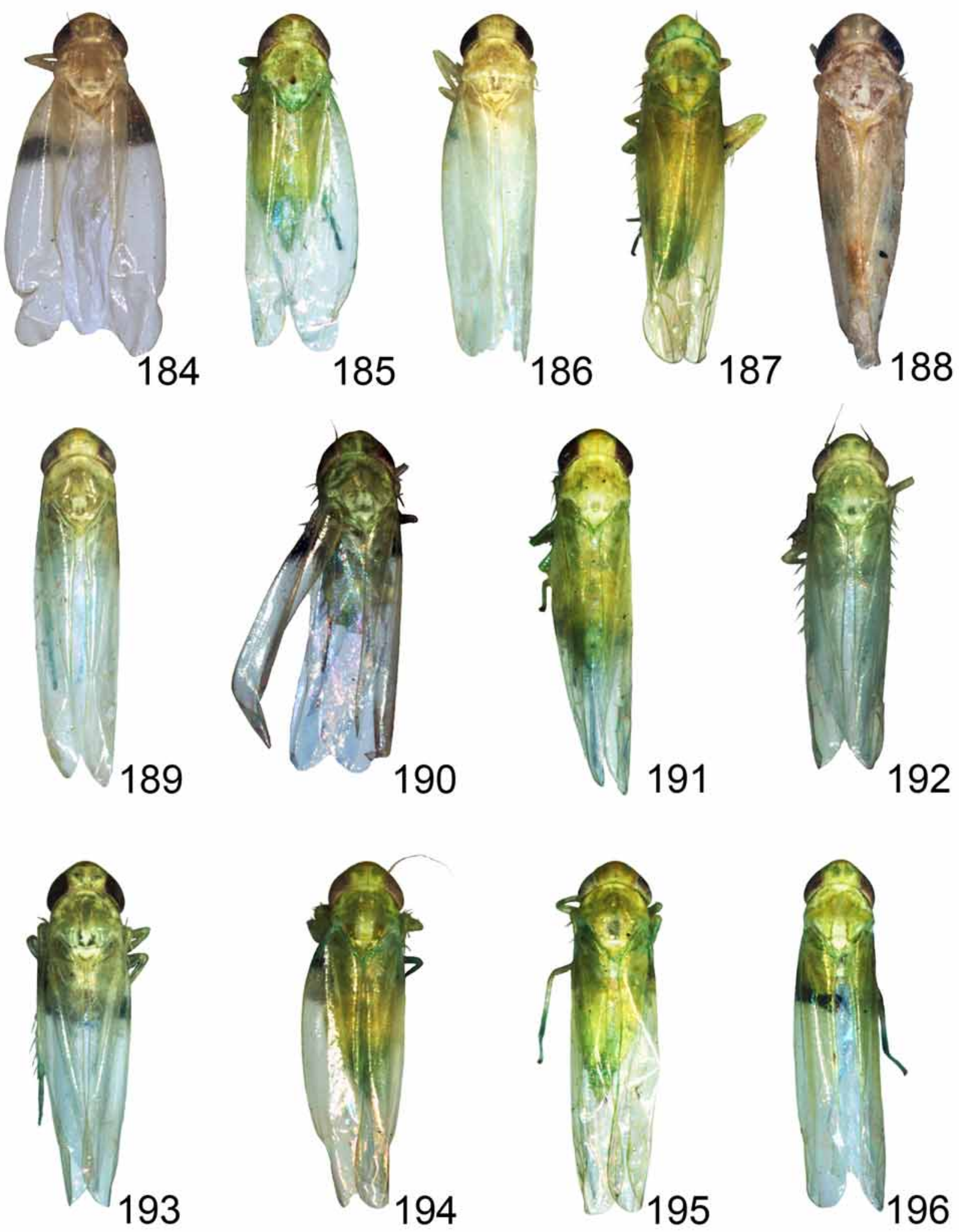

FIGURES 184-196. Whole body, dorsal view. 184, Empoasca (Distantasca) terminalis Distant; 185, Empoasca (Distantasca) faciata (Dworakowska); 186, Empoasca (Distantasca) riora Dworakowska; 187, Empoasca (Distantasca) tna Dworakowska; 188, Empoasca (Distantasca) latava Dworakowska; 189, Empoasca (Distantasca) rokasa Dworakowska; 190, Empoasca (Distantasca) atika Dworakowska; 191, Empoasca (Distantasca) tiaca Dworakowska; 192, Empoasca (Distantasca) bulbosa Dworakowska; 193, Empoasca (Distantasca) paraterminalis Qin \& Zhang; 194, Empoasca (Distantasca) serratipenis Qin \& Zhang; 195, Empoasca (Distantasca) tuberculata Zhang \& Liu sp. n.; 196, Empoasca (Distantasca) latibasis Zhang \& Liu sp. n. 

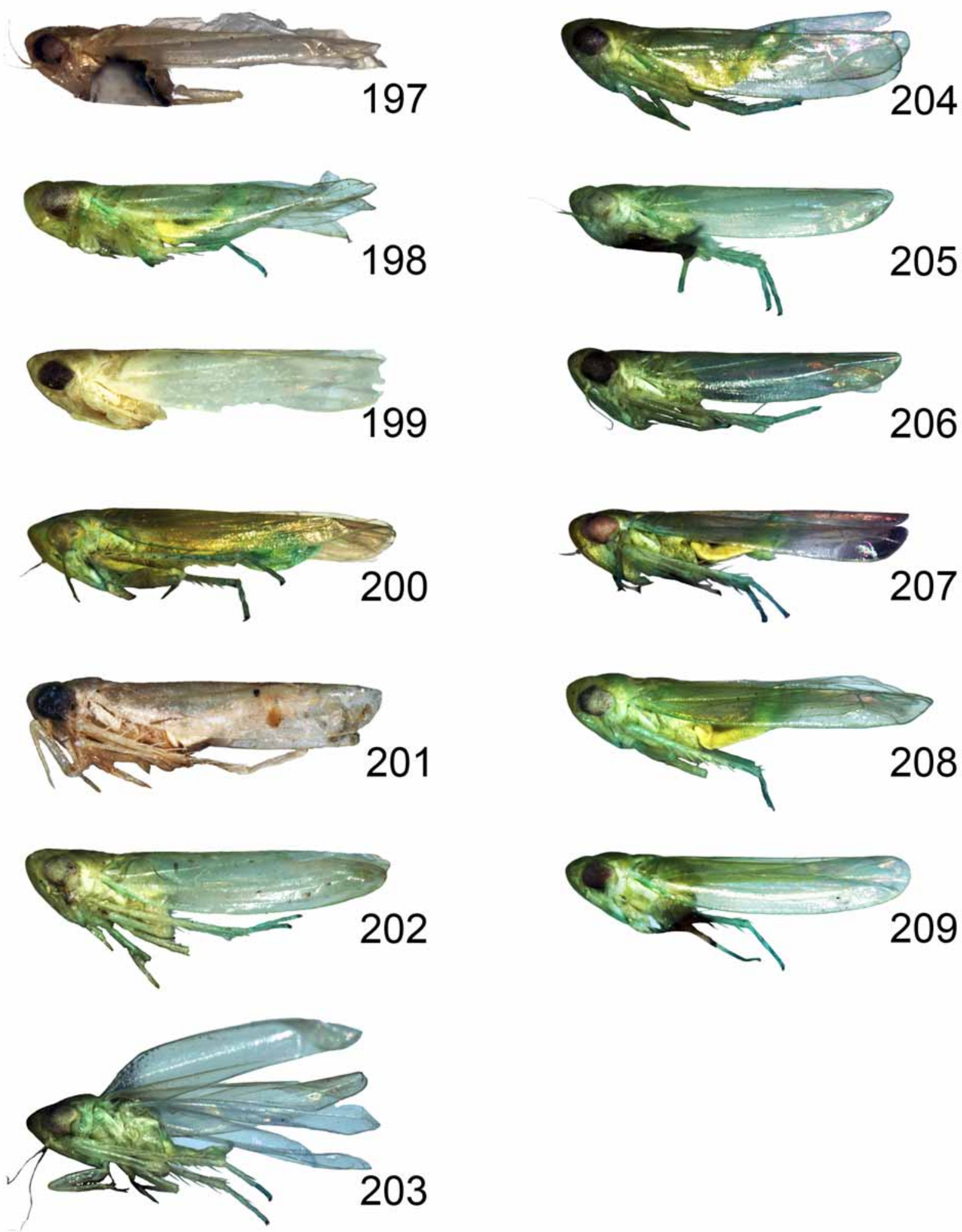

FIGURES 197-209. Whole body, lateral view. 197, Empoasca (Distantasca) terminalis Distant; 198, Empoasca (Distantasca) faciata (Dworakowska); 199, Empoasca (Distantasca) riora Dworakowska; 200, Empoasca (Distantasca) tna Dworakowska; 201, Empoasca (Distantasca) latava Dworakowska; 202, Empoasca (Distantasca) rokasa Dworakowska; 203, Empoasca (Distantasca) atika Dworakowska; 204, Empoasca (Distantasca) tiaca Dworakowska; 205, Empoasca (Distantasca) bulbosa Dworakowska; 206, Empoasca (Distantasca) paraterminalis Qin \& Zhang; 207, Empoasca (Distantasca) serratipenis Qin \& Zhang; 208, Empoasca (Distantasca) tuberculata Zhang \& Liu sp. n.; 209, Empoasca (Distantasca) latibasis Zhang \& Liu sp. n. 
Empoasca (Distantasca) bulbosa Dworakowska, 1994: 102; Qin \& Zhang, 2007: 191.

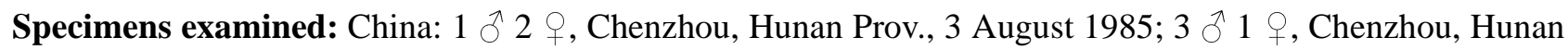
Prov., 16 August 1985; above all were collected by Zhang Yalin; 1 ऽ, Sangang in Wuyishan, Fujian Prov., 23 August 1988, coll. Yang Zhongqi; 1 $\ 1$, Tianpingshan in Sangzhi, Hunan Prov., 14 August 2001, coll. Sun Qiang.

Distribution: China (Hunan, Fujian); Sikkim.

Notes: In some specimens, the anal tube appendage is bifid, differing from the unbranched appendage of the holytype.

Empoasca (Distantasca) paraterminalis Qin \& Zhang, 2007

(Figs. 140-148, 167, 180, 193, 206)

Empoasca (Distantasca) paraterminalis Qin \& Zhang, 2007: 187-188.

Specimens examined: China: 1 (Holotype), Sanchahe in Mengyang, Yunnan Prov., 6 June 1991, coll. Tian Rungang, Cai Wanzhi, Wang Yinglun.

Distribution: China (Yunnan).

Empoasca (Distantasca) serratipenis Qin \& Zhang, 2007

(Figs. 149-157, 168, 181, 194, 207)

Empoasca (Distantasca) serratipenis Qin \& Zhang, 2007: 187-190.

Specimens examined: China: $1 \overbrace{}^{\top}$ (Holotype), Mengla, Yunnan Prov., 6 July 1999, 700m, coll. Qin Daozheng. Distribution: China (Yunnan).

Empoasca (Distantasca) tuberculata Zhang \& Liu sp. n.

(Figs. 118-127, 169, 182, 195, 208)

Type material. Holotype: $\widehat{\jmath}$ (NWAFU), Mengyuan, Yunnan Prov., China, 11 Nov. 1999, coll. Dworakowska. Paratypes, $1 \hat{\partial}$, same data as holotype; $1 \hat{\partial}$, Mengyuan, Yunnan Prov., China, 11 Nov. 1999, coll. Qin Daozheng.

Length. Male: $3.2-3.4 \mathrm{~mm}$.

Ground colour of body yellowish-green. Crown with light yellow or cream markings on each side of coronal suture. Eyes blackish-brown. Pronotum with irregular patches on anterior margin of pronotum and behind eyes. Forewing and hind wing semitransparent. Abdomen yellow. Legs light yellow to gold.

Abdominal apodemes subparallel-sided, reaching base of $5^{\text {th }}$ segment (Fig. 123). Male pygofer slightly narrowing caudad, ornamented with 12-16 stout setae on each side of pygofer lobe; ventral pygofer appendage slightly curved dorsocaudad, exceeding caudal margin of lobe (Fig. 118). Anal appendage long, broad at base, tapering to pointed apex with some irregular teeth (Fig. 125). Subgenital plate sinuate, with numerous macrosetae and fine setae scattered irregularly; inner margin with 22-27 short microsetae on apical half and two bands of long, hair-like setae on lateral surface-one near mid-length of plate and one just before apex, often separated by few thin but short setae (Fig. 127). Paramere with 8 teeth on dentifer, 4-6 setae subapically (Fig. 126). Shaft as long as preatrium, broad at base in lateral view, gradually tapering and curved 
at basal 2/5, shaft subbasally with pair of processes directed caudodorsad; each process with teeth-like or papillose tubercles arranged irregularly at apex, gonopore subterminal on ventral side (Figs. 121, 122). Connective broad, with posterior margin deeply emarginate medially (Fig. 124).

Etymology. The species name is derived from the Latin word "tuberculata", which refers to the tooth-like or papillose tubercles on the apices of the long processes of the aedeagus.

Distribution. Known only from the type locality in Yunnan Province (SW China).

Remarks. This new species is similar to Empoasca (Distantasca) serratipenis Qin \& Zhang, 2007, but can be distinguished from the latter by the following characters: aedeagal shaft fairly long, with pair of distally serrate processes arising near base.

\section{Empoasca (Distantasca) latibasis Zhang \& Liu sp. n.}

(Figs. 128-139, 170, 183, 196, 209)

Type material. Holotype: $\widehat{~(N W A F U), ~ M u n a i, ~ Y u n n a n ~ P r o v ., ~ C h i n a, ~} 2$ Dec. 2004, 1100m, coll. Dworakowska. Paratypes: 1 त 1 , same data as holotype; 1 , Munai, Yunnan Prov., China, 2 Dec. 2004, 1100m, coll. Qin Daozheng.

Length. Male: $3.2-3.4 \mathrm{~mm}$, female: $3.3 \mathrm{~mm}$.

Ground colour of body green-yellowish. Crown with pale green patch on each side of coronal suture, in some specimens with narrow brown line along medial suture. Eyes black-brown. Pronotum broad, with irregular patches of cream-yellowish on anterior margin. Forewing semitransparent with light yellow-greenish tint, hind wing transparent. Abdomen yellow. Legs light yellow to gold.

Abdominal apodemes long, reaching middle of $5^{\text {th }}$ segment, subparallel-sided to rounded apices, some specimens (including holotype) with small triangular projection extended ventrad from $4^{\text {th }}$ segment margin on either side of midline, apodeme with lightly sclerotized spot just above each projection (Fig. 134). Male pygofer slightly narrowing caudad, ornamented with 11-14 rigid setae on each side of pygofer lobe (Fig. 128); ventral pygofer appendage slightly curved dorsocaudad, exceeding caudal margin of lobe (Fig. 129). Anal appendage stout, strongly constricted and tapered to acute apex, with big tooth subapically (Fig. 137), in one specimen anal appendage slightly different (Fig. 136). Subgenital plate sinuate, with numerous macrosetae and fine setae in somewhat irregular arrangement; inner margin with 22-29 short microsetae on apical half and two bands of long, hair-like setae on lateral surface-one near mid-length of plate and one just before apex (Fig. 139). Paramere with 7 teeth on dentifer, 4-8 setae subapically (Fig. 138). In posteroventral view, aedeagus relatively thin, expanding gradually to atrium, shaft thin and subparallel-sided, apex with lateral processes directed caudoventrad in profile, gonopore subterminal on ventral surface. Connective broad, with caudal margin deeply emarginate medially (Fig. 135).

Etymology. The species name is derived from the Latin words "latus" or broad and the Greek "basis" or foundation and refers to the very strong base of the anal appendage.

Distribution. Known only from the type locality in Yunnan Province (SW China).

Remarks. The new species is similar to Empoasca (Distantasca) paraterminalis Qin \& Zhang, 2007, but differs in the length of the pygofer appendage which is shorter than the style; in the aedeagus, with lateral processes apically directed caudoventrad in profile, and in not having two pairs of subbasal appendages.

\section{Acknowledgement}

We are very grateful to Dr. Sterling Southern, Department of Entomology, North Carolina University for revising the manuscript and suggesting improvements. This study was supported by The National Natural Science Foundation of China (30770262) and Basic Science and Technology Project of the Ministry of Science and Technology of China (2006FY120100). 


\section{References}

Distant, W.L. (1918) The fauna of British India, including Ceylon and Burma, Rhynchota. Vol. 7. Homoptera: Appendix, Heteroptera: Addenda, 7, i-vii, 1-210.

Dworakowska, I. (1972) On some Oriental and Ethiopian genera of Empoascini (Auchenorrhyncha: Cicadellidae: Typhlocybinae). Bulletin de l'Academie Polonaise des Sciences. Serie des Sciences Biologiques, 20 (1), $25-34$.

Dworakowska, I. \& Viraktamath, C.A. (1975) On some Typhlocybinae from India (Auchenorrhyncha, Cicadellidae). Bulletin de l'Academie Polonaise des Sciences. Serie des Sciences Biologiques, 23 (8), 521-530.

Dworakowska, I. (1976), On some Oriental and Ethiopian Typhlocybinae (Homoptera: Auchenorrhyncha: Cicadellidae). Reichenbachia, 16 (1), 1-51.

Dworakowska, I. (1977) On some Typhlocybinae from Vietnam (Homoptera: Cicadellidae). Folia Entomologica Hungarica, 30 (2), 9-47.

Dworakowska, I. (1980) On some Typhlocybinae from India (Homoptera: Auchenorrhyncha: Cicadellidae). Entomologische Abhandlungen, 43 (8), 151-201.

Dworakowska, I. (1981) On some Typhlocybinae from India, Sri Lanka and Nepal (Homoptera: Auchenorrhyncha: Cicadellidae). Entomologische Abhandlungen und Berichte aus dem Staatlichen Museum fur Tierkunde in Dresden, 44 (8), 153-202.

Dworakowska, I. (1982) Empoascini of Japan, Korea and north-east part of China (Homoptera: Auchenorrhyncha: Cicadellidae: Typhlocybinae). Reichenbachia, 20 (1), 33-57.

Dworakowska, I. (1993) Remarks on Alebra Fieb. and Eastern Hemisphere Alerini ( Auchenorrhyncha: Cicadellidae: Typhlocybinae). Entomotaxonomia, 15 (2): 91-121.

Dworakowska, I. (1994) Typhlocybinae (Auchenorrhyncha: Cicadellidae) of Sikkim, a preliminary survey. Folia Entomologica Hungarica, 55, 93-215.

Linnavaori, R. (1960) Insects of Micronesia. Homoptera: Cicadellidae. Honolulu, Bishop Museum, 6 (5), $231-344$.

Oman, P.W., Knight, W.J. \& Nielson, M.W. (1990) Leafhoppers (Cicadellidae): A bibliography, generic checklist and index to the world literature 1956-1985. C.A.B International Institute of Entomology, 1-368.

Qin, D.Z. \& Zhang, Y.L. (2007) A taxonomic study on the subgenus Empoasca (Distantasca) Dworakowska (Hemiptera: Cicadellidae: Typhlocybinae: Empoascini) from China. The Pan-Pacific Entomologist, 83 (3), 185-192.

Zhang, Y.L. (1990) A taxonomic study of Chinese Cicadellidae (Homoptera). Tianze Eldonejo. Yangling, Shaanxi, China. $218 \mathrm{pp}$. 